\title{
Allosteric Modulation of the CB1 Cannabinoid Receptor by Cannabidiol-A Molecular Modeling Study of the N-Terminal Domain and the Allosteric-Orthosteric Coupling
}

\author{
Jakub Jakowiecki ${ }^{1, *(1)}$, Renata Abel ${ }^{2,3}\left(\mathbb{D}\right.$, Urszula Orzeł ${ }^{1}$, Paweł Pasznik ${ }^{1}$, Robert Preissner ${ }^{2}(\mathbb{D})$ \\ and Sławomir Filipek $1, *$ D \\ 1 Biological and Chemical Research Centre, Faculty of Chemistry, University of Warsaw, 02093 Warsaw, Poland; \\ u.orzel@student.uw.edu.pl (U.O.); p.pasznik@gmail.com (P.P.) \\ 2 Structural Bioinformatics Group, Institute for Physiology, Charité-University Medicine Berlin, \\ 10115 Berlin, Germany; renata.abel@charite.de (R.A.); robert.preissner@charite.de (R.P.) \\ 3 Department of Pharmaceutical Biology and Botany, Wroclaw Medical University, 50556 Wroclaw, Poland \\ * Correspondence: jjakowiecki@chem.uw.edu.pl (J.J.); sfilipek@chem.uw.edu.pl (S.F.)
}

check for

updates

Citation: Jakowiecki, J.; Abel, R.; Orzeł, U.; Pasznik, P.; Preissner, R.; Filipek, S. Allosteric Modulation of the CB1 Cannabinoid Receptor by Cannabidiol-A Molecular Modeling Study of the N-Terminal Domain and the Allosteric-Orthosteric Coupling. Molecules 2021, 26, 2456. https:// doi.org/10.3390/molecules26092456

Academic Editor: Alessandra Bisi

Received: 7 March 2021

Accepted: 19 April 2021

Published: 23 April 2021

Publisher's Note: MDPI stays neutra with regard to jurisdictional claims in published maps and institutional affiliations.

Copyright: (c) 2021 by the authors. Licensee MDPI, Basel, Switzerland. This article is an open access article distributed under the terms and conditions of the Creative Commons Attribution (CC BY) license (https:// creativecommons.org/licenses/by/ $4.0 /)$.

\begin{abstract}
The $\mathrm{CB}_{1}$ cannabinoid receptor $\left(\mathrm{CB}_{1} \mathrm{R}\right)$ contains one of the longest $\mathrm{N}$ termini among class $\mathrm{AG}$ protein-coupled receptors. Mutagenesis studies suggest that the allosteric binding site of cannabidiol (CBD) involves residues from the $\mathrm{N}$ terminal domain. In order to study the allosteric binding of CBD to $\mathrm{CB}_{1} \mathrm{R}$ we modeled the whole $\mathrm{N}$-terminus of this receptor using the replica exchange molecular dynamics with solute tempering (REST2) approach. Then, the obtained structures of $\mathrm{CB}_{1} \mathrm{R}$ with the $\mathrm{N}$ terminus were used for ligand docking. A natural cannabinoid receptor agonist, $\Delta^{9}-\mathrm{THC}$, was docked to the orthosteric site and a negative allosteric modulator, CBD, to the allosteric site positioned between extracellular ends of helices TM1 and TM2. The molecular dynamics simulations were then performed for $\mathrm{CB}_{1} \mathrm{R}$ with ligands: (i) $\mathrm{CBD}$ together with THC, and (ii) THC-only. Analyses of the differences in the residue-residue interaction patterns between those two cases allowed us to elucidate the allosteric network responsible for the modulation of the $\mathrm{CB}_{1} \mathrm{R}$ by $\mathrm{CBD}$. In addition, we identified the changes in the orthosteric binding mode of $\Delta^{9}$-THC, as well as the changes in its binding energy, caused by the CBD allosteric binding. We have also found that the presence of a complete $\mathrm{N}$-terminal domain is essential for a stable binding of $\mathrm{CBD}$ in the allosteric site of $\mathrm{CB}_{1} \mathrm{R}$ as well as for the allosteric-orthosteric coupling mechanism.
\end{abstract}

Keywords: CB1; cannabinoid receptor; GPCRs; allosteric modulation; CBD; THC; interaction fingerprints; molecular dynamics; REST2

\section{Introduction}

The cannabinoid $C_{1}$ receptor is the most abundant $G$ protein-coupled receptor (GPCR) in the human central nervous system [1] and, together with $\mathrm{CB}_{2}$ receptor, is a part of the endocannabinoid system. Cannabinoid receptors participate in many biological processes, including mood regulation, appetite and lipid metabolism, cognitive functions, nociception, as well as cell growth and proliferation [2]. In particular, $C_{1} R$ is a very attractive pharmacological target for treatment of many disorders [3], such as: multiple sclerosis, Parkinson's disease, Huntington's disease, epilepsy, pain, appetite deregulation and obesity [4,5]. Before the discovery of the allosteric binding site(s) in $C_{1} R$, designing new ligands for this receptor was solely based on the orthosteric binding site [6]. A long list of $\mathrm{CB}_{1} \mathrm{R}$ ligands including endocannabinoids, phytocannabinoids, and synthetic compounds have been discovered and developed over the last 20 years. In 2005, it was discovered that the $\mathrm{CB}_{1}$ receptor contains allosteric binding site(s) that can be targeted by small molecule ligands [7,8]. 


\subsection{Advantages of Allosteric over Orthosteric Ligands of $C B_{1} R$}

Considering the psychomimetic effects commonly associated with $\mathrm{CB}_{1} \mathrm{R}$ agonists and depressant effects associated with $\mathrm{CB}_{1}$ antagonists/inverse agonists, allosteric modulation may offer an alternative approach to attain potential therapeutic benefits while avoiding the unwanted side effects of orthosteric ligands [7]. Allosteric modulators do not possess intrinsic efficacy, but instead they enhance or diminish the receptor's response to orthosteric ligands. In the absence of any exogenous $\mathrm{CB}_{1} \mathrm{R}$ orthosteric ligands the maximum effect of an allosteric modulator is determined by the level of endocannabinoids, such as anandamide (AEA) and 2-arachidonylglycerol (2-AG) (Figure 1) [9,10]. While the exogenous orthosteric ligands may produce adverse effects through long term over-activation or down-regulation of a receptor $[9,10]$, the allosteric modulators of $\mathrm{CB}_{1} \mathrm{R}$ may not cause those undesirable effects since their efficacy depends on the presence of orthosteric ligands. Endogenous orthosteric ligands are released on demand and removed by enzymatic degradation (i.e., processed by fatty acid amide hydrolase (FAAH) [11]) or cellular reuptake when no longer needed. Therefore, the allosteric modulators allow for a tempering of cannabinoid receptor signalling without desensitization, tolerance or dependence [12]. In addition, the GPCR allosteric binding sites are often less conserved than orthosteric sites, thus the allosteric ligands have potentially greater specificity for particular receptor types and subtypes [7].
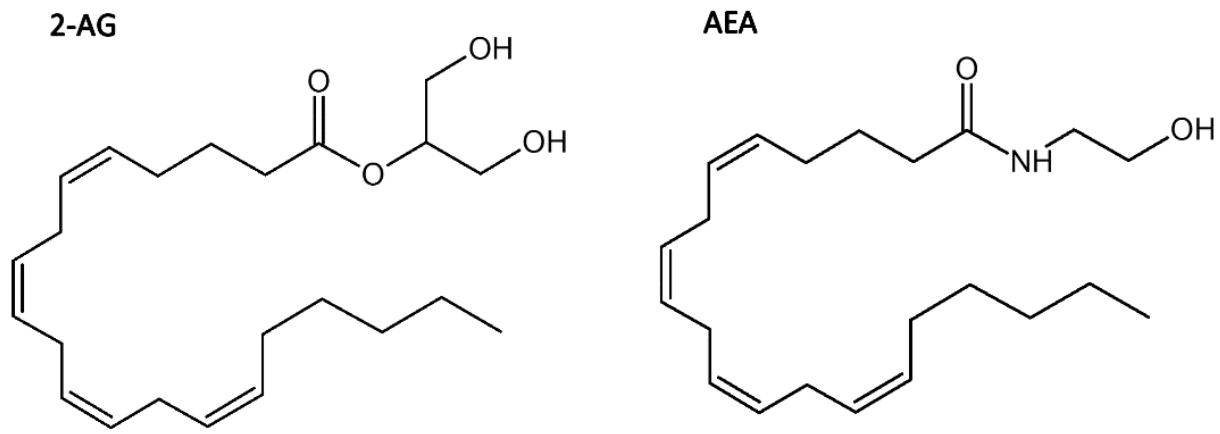

Figure 1. Chemical structures of endocannabinoids: 2-arachidonylglycerol (2-AG) and anandamide (AEA).

The allosteric ligands can be classified based on their effects on receptor signalling. There are positive allosteric modulators (PAMs), which enhance the orthosteric agonists signalling efficacy (independently of their effect on the biding affinity of the orthosteric ligand, which can be either positive, negative or null), and the negative allosteric modulators (NAMs), which attenuate the orthosteric agonists signalling. Apart from the modulators there are also different kinds of allosteric ligands, such as allosteric agonists capable of enhancing receptor activity even in the absence of an orthosteric agonist, allosteric antagonists that can supress receptor activation induced by orthosteric agonists (in a non-competitive manner) and, finally, neutral allosteric ligands (NALs), which bind to the allosteric binding sites without affecting receptor activity and affinity [13-15]. The activity of an allosteric modulator depends on the orthosteric probe used. Both the change in orthosteric ligand binding affinity and the magnitude of the allosteric modulation effect will vary between different orthosteric ligands [10,16].

\subsection{Cannabidiol-Its Properties and the Binding Site}

Cannabidiol (CBD) is one of the major phytocannabinoids present in Cannabis plants. Either alone or in a mixture with $\Delta^{9}$-tetrahydrocannabinol $\left(\Delta^{9}\right.$-THC) it is being intensively studied as a safe and efficacious alternative for the treatment of various medical conditions such as cancer, multiple sclerosis, Parkinson's (PD) and Alzheimer's disease (AD). It has been recently approved in some countries for the treatment of some specific types of treatment-resistant childhood epilepsy [17]. The properties of CBD including their possible biological targets and docking studies were recently reviewed in [18]. 
Recently, it has been also proposed that CBD can have potential usage as a support drug for the treatment of COVID-19 disease [19-22]. There are several mechanisms (both $\mathrm{CB}_{1} \mathrm{R} / \mathrm{CB}_{2} \mathrm{R}$ dependent and independent) that substantiate the potential benefits of CBD usage in anti-COVID-19 therapy. Firstly, the well-known anti-inflammatory and immunomodulatory activity of CBD that can inhibit an excessive immunological response leading to a cytokine storm, which is a direct cause of severe lung injury observed in many COVID-19 patients [23]. It has been shown that CBD can ameliorate the symptoms of acute respiratory distress syndrome in mice through the upregulation of apelin [21] and studies on humans have shown a reduction of some pro-inflammatory monocytes production and of CD4 and CD8 T-cells activation frequencies in response to cannabinoids [20,24]. Secondly, CBD can possibly attenuate the crucial routes of SARS-CoV2 infection in humans since cannabis extract with a high CBD content was able to down-regulate the expression of two receptors needed for SARS-CoV2 entry into the cell: the angiotensin-converting enzyme 2 (ACE2) and the transmembrane serine protease 2 (TMPRSS2) [19,25]. Thirdly, $\mathrm{CBD}$ is a PPAR $\gamma$ (peroxisome proliferator-activated receptor- $\gamma$ ) agonist so it can display a direct antiviral activity. In addition, PPAR $\gamma$ agonists regulate fibroblast/myofibroblast activation and thus they can inhibit the development of pulmonary fibrosis [19]. Despite the fact that some of those reports sound very promising more data are needed to justify the use of CBD in the treatment of COVID-19.

Although CBD is an isomer of THC (Figure 1) it has no psychoactive properties. In fact, it even suppresses some of the undesired side effects induced by $\Delta^{9}$-THC such as intoxication, sedation and tachycardia while attenuating its medical effects such as analgesic, anti-emetic, and anti-carcinogenic effects [26]. Together with terpenoids and other phytocannabinoids present in cannabis CBD contributes to the synergistic entourage effect [27]. $C B D$ binds to a distinct binding site than the orthosteric ligands since it antagonises $C_{1} R$ orthosteric agonists at concentrations far below its reported affinity [28]. The NAM activity of CBD was also demonstrated by Lapraire et al. [10] who showed that CBD reduces the potency and efficacy of $\Delta^{9}$-THC and 2-AG in a non-competitive manner. Their results also indicate that the $\mathrm{CBD}$ allosteric binding site is located within the $\mathrm{CB}_{1} \mathrm{R} N$-terminal domain and is particularly close to residues C98 and C107, which form a disulfide bond. They performed mutagenesis and BRET studies demonstrating that the C98A mutation as well as the C107A mutation led to a 50\% loss of CBD NAM activity, versus both agonists, $\Delta^{9}$-THC and 2-AG, when compared to $\mathrm{WT} \mathrm{CB}_{1} \mathrm{R}$. Interestingly, other mutations at those residues, C98S and C107S, caused no change in CBD NAM activity [10], which suggests that other interactions can substitute for the disulfide bridge and stabilize the local structure of $C B_{1} R$. Presence of the Cys98-Cys107 bond also affects the allosteric activity of ORG27569 and PSNCBAM-1 (Figure 2) [29] and it is very likely that CBD binds to the same allosteric site as those two compounds. However, in contrast to ORG27569 and PSNCBAM-1, for which the ligand cooperativity is positive for agonists and negative for antagonists and inverse agonists [8,30], CBD displayed a negative cooperativity for all tested ligands [10].
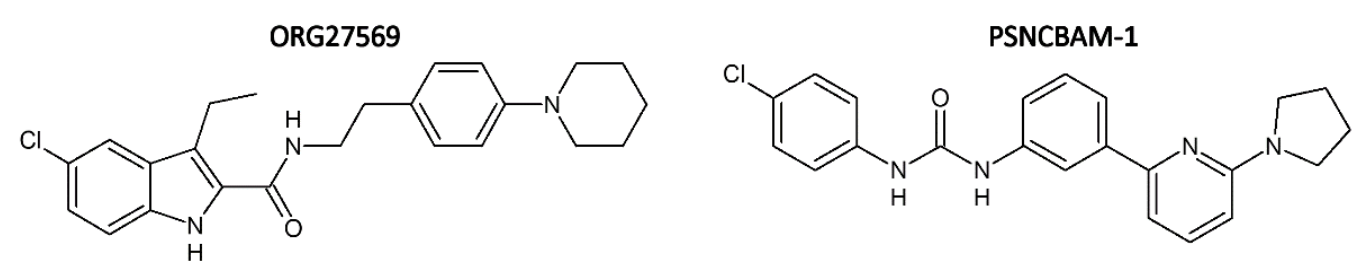

Figure 2. Chemical structures of $\mathrm{CB}_{1} \mathrm{R}$ allosteric ligands ORG27569 and PSNCBAM-1.

\subsection{The Importance of N-Terminus for Allosteric and Orthosteric Binding}

We have concluded that the role of the $\mathrm{CB}_{1} \mathrm{R} N$-terminal domain in both allosteric and orthosteric ligand binding was significantly underestimated during recent years, and for this reason we decided to determine its structure as a whole, dedicating a special effort to modeling of the membrane-proximal region (MPR) and the allosteric binding site of 
CBD. The main inspiration for our project was the experimental work of Lapraire et al. on $\mathrm{CBD}$ binding [10]. In our research we have focused on exploring the $\mathrm{CB}_{1}$ receptor allosteric binding site (allosteric site 1) located between the extracellular ends of helices I and II (colored in blue and cyan in Figure 3, respectively). The three allosteric sites (Figure 1) were proposed by Sabatucci et al. [31] who found that CBD very likely binds to the allosteric site 1.

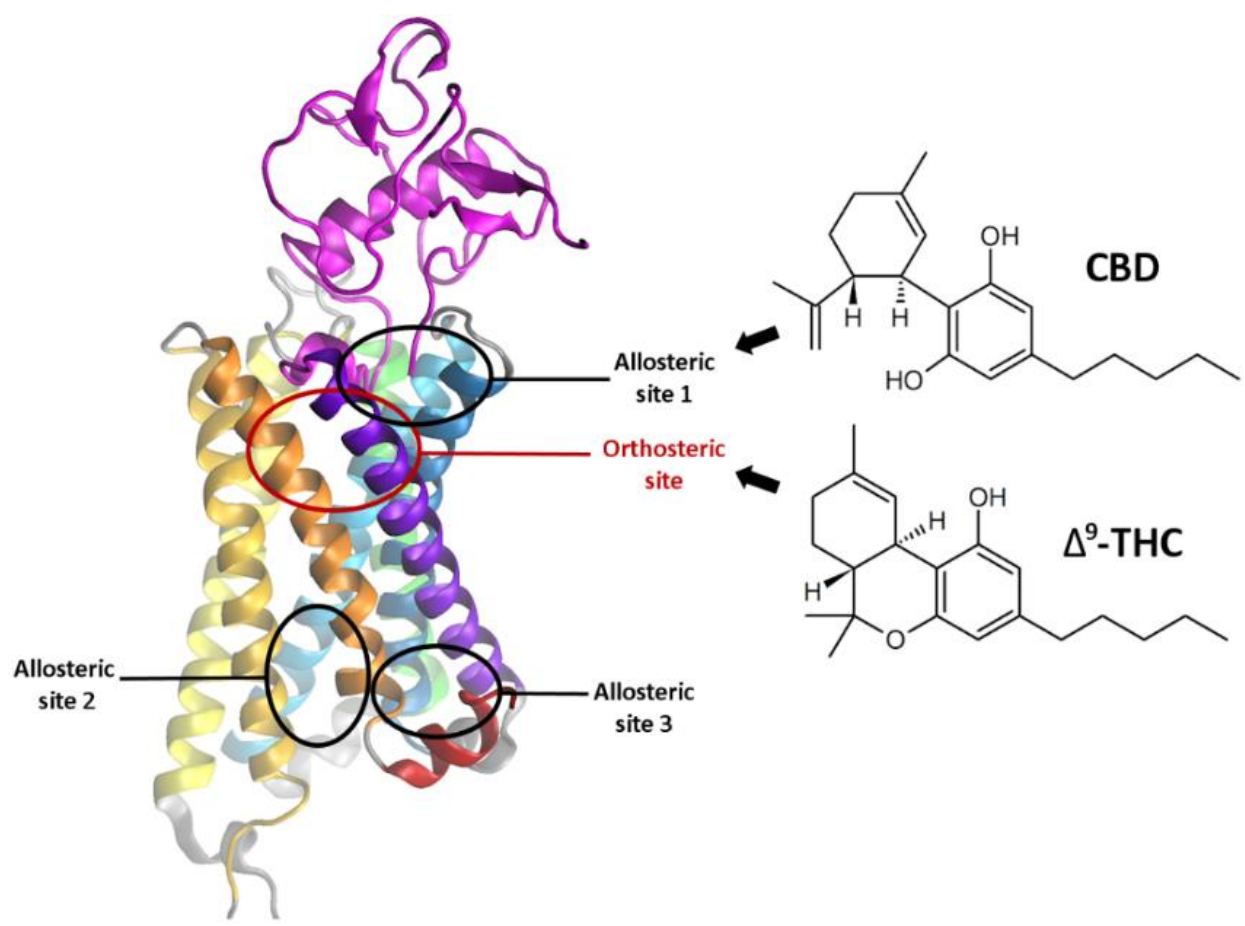

Figure 3. CB1 cannabinoid receptor with modelled N-terminus and the locations of orthosteric and allosteric binding sites. Cannabidiol (CBD)—an allosteric ligand, and $\Delta 9$-tetrahydrocannabinol $\left(\Delta^{9}-\mathrm{THC}\right)$ - an orthosteric ligand of CB1R. According to Lapraire et al. and Sabatucci et al. CBD very likely binds to the allosteric site $1[10,31]$.

Sabatucci et al. performed docking studies in order to map the allosteric binding sites of $C_{1} R$ [31]. However, as they point out themselves, for docking they used the crystal structures 5TGZ and 5U09, which lack most of the N-terminal residues, including C98, which has been shown to have an impact on allosteric modulation of the $\mathrm{CB}_{1}$ receptor $[10,29]$. Besides, they did not perform any molecular dynamics simulations that would confirm the stability of CBD binding in the allosteric site 1. A study on CBD allosteric binding that employed MD simulations has been done by Chung et al. [32]. They have reported an outward rotation of TM1 and TM2 upon CBD binding to the allosteric site 1, resulting in the expansion of the orthosteric binding site. They also revealed that CBD hydroxyl group formed interactions with D104 (which is in agreement with our results) while its alkyl chain formed hydrophobic contacts with F102, I105, I267 and F268. However, it should be pointed out that they used very short MD simulations, 25-50 ns, which were very likely insufficient for verification of CBD binding mode stability. Besides, those studies relied on crystal/cryo-EM structures supplemented with a small, proximal part of N-terminus only, without considering the influence of the complete N-terminal domain on the allosteric binding.

There is a large difference between lengths of $\mathrm{N}$-termini of $\mathrm{CB}_{1}$ (111 residues) and $\mathrm{CB}_{2}$ (28 residues) receptors. We have done a comparison of N-termini of class A GPCRs excluding odorant receptors (Figure 4). The $\mathrm{CB}_{1} \mathrm{R}$ N-terminus is one of 14 longest $\mathrm{N}$-termini of those receptors while for $\mathrm{CB}_{2} \mathrm{R}$ it has the most typical length. Because of this difference the allosteric-orthosteric coupling in both cannabinoid receptors is probably much different. 

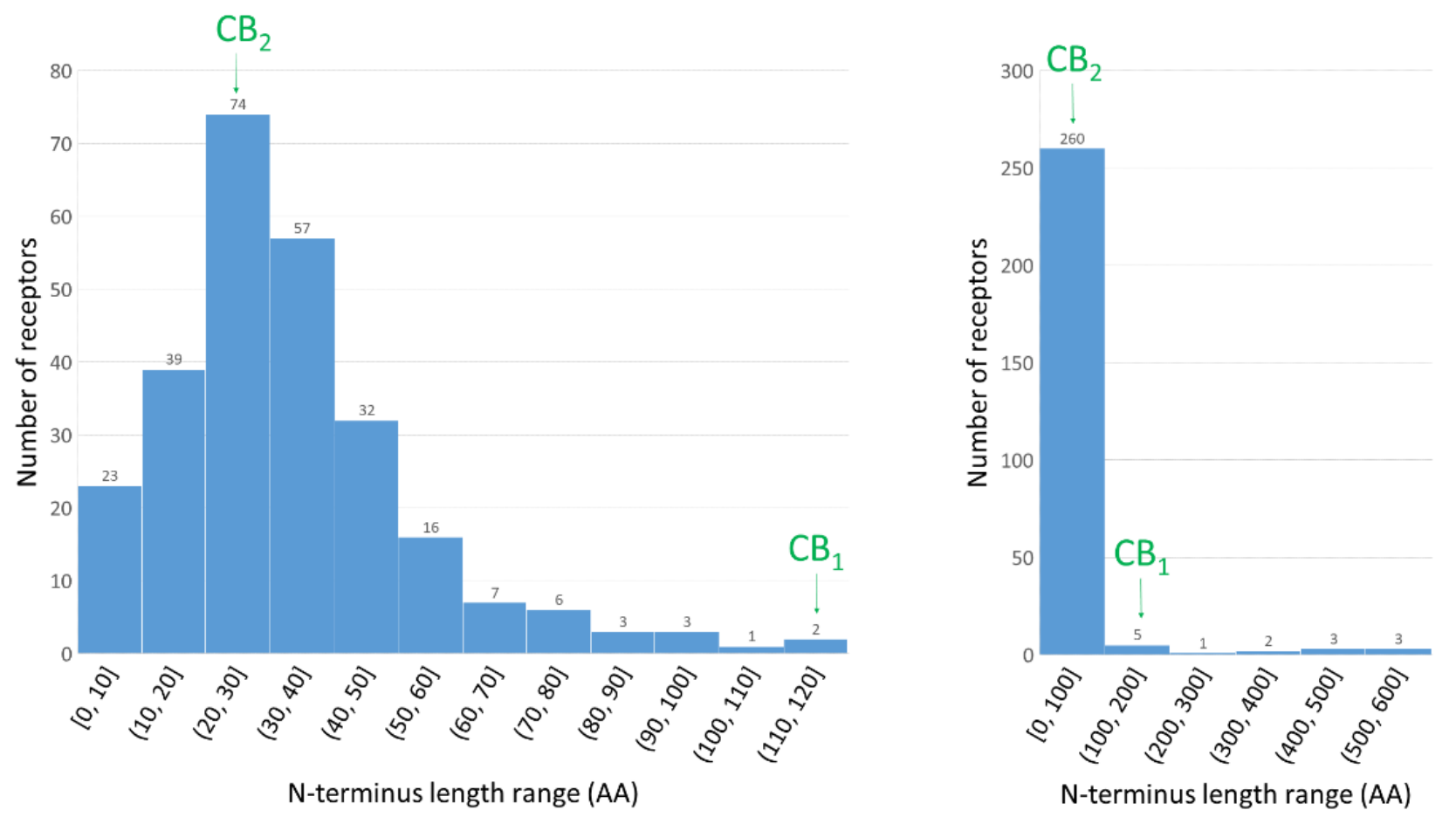

Figure 4. Distribution of N-terminus lengths within class A of GPCRs. On left the histogram with 10 amino acid interval; on right the histogram for all class A GPCR, without odorant receptors, with 100 amino acid interval. Number of receptors in each range is specified above each bar. The ranges are given in parenthesis which are open from left side. Based on GPCRdb data [33].

In our studies we have modeled the structure of the whole $\mathrm{N}$-terminal domain of $\mathrm{CB}_{1} \mathrm{R}$, docked $\mathrm{CBD}$ and THC to their binding sites, and run a set of long molecular dynamics (MD) simulations, $10 \times 1 \mu \mathrm{s}$, to obtain statistically valid results. All the above simulations started from the same structure of $\mathrm{CB}_{1} \mathrm{R}$ with a complete $\mathrm{N}$-terminus and THC bound to the orthosteric site. CBD was bound to the allosteric site 1 in five simulations, while in the other five simulations the allosteric site was empty. Analysis of the residue contact network and, especially, the differences in the interaction patterns between those two sets of trajectories, revealed interactions that are crucial for the allosteric-orthosteric coupling, called here the 'allosteric network'. We also investigated the relevance of $\mathrm{CB}_{1} \mathrm{R} N$-terminus for $\mathrm{CBD}$ allosteric binding by gradually truncating the $\mathrm{N}$-terminus starting from its first residue and analyzing the changes in its stability and in the ligand binding energy. All performed MD simulations (including REST2 simulations) took over $150 \mu$ s (Table 1).

Table 1. The MD simulations performed in this work.

\begin{tabular}{|c|c|c|c|c|}
\hline MD Simulation Type & Average Length $[\mu \mathrm{s}]$ & Repeats & REST2 Replicas & Cumulative Length $[\mu \mathrm{s}]$ \\
\hline $\mathrm{CB}_{1} \mathrm{R}$ with MPR & 0.25 & 8 & 1 & 2 \\
\hline REST2 of $\mathrm{CB}_{1} \mathrm{R}$ with complete $\mathrm{N}$-terminus & 1.2 & 6 & 16 & 115.2 \\
\hline $\mathrm{CB}_{1} \mathrm{R}$ with CBD-THC (for contact map) & 1 & 5 & 1 & 5 \\
\hline $\mathrm{CB}_{1} \mathrm{R}$ with THC-only (for contact map) & 1 & 5 & 1 & 5 \\
\hline $\begin{array}{c}\mathrm{CB}_{1} \mathrm{R} \text { N-terminus stability corroboration } \\
\text { (with and without } \mathrm{CBD} \text { ) }\end{array}$ & $2 \times 3$ & 3 & 1 & 18 \\
\hline $\mathrm{CB}_{1} \mathrm{R}$ with truncated $\mathrm{N}$-term (mutation studies) & $3 \times 1$ & 2 & 1 & 6 \\
\hline Total & & & & 151.2 \\
\hline
\end{tabular}




\section{Results and Discussion}

\subsection{Construction of the $N$-Terminus of $C B_{1} R$}

The so called membrane-proximal region (MPR) of cannabinoid receptor ( residues 90-110) has been proved to be crucial not only for the allosteric ligands binding, but also for binding of the orthosteric ligands [29]. According to Laprairie et al. [10] the 96-112 fragment (stabilized by the C98-C107 disulfide bond) is essential for the NAM activity of CBD. Therefore, we started with modeling of this small fragment. The task was made easier by the fact that the residues 100-112 were already present in the crystal structure of $\mathrm{CB}_{1} \mathrm{R}$ (PDB ID: 5U09 [34]), so only the 4 residues (96-99) were added. We chose this particular crystal structure because it had the longest $\mathrm{N}$-terminal part from all available experimental structures of that receptor. Also, it is very likely that this structure contains the disulfide bridge C98-C107 since the first residue occurring in the crystallized protein is G89, although the visible part of the structure starts from residue E100. Therefore, we assume that the geometry of the 100-112 fragment is the most correct among all available $\mathrm{CB}_{1} \mathrm{R}$ experimental structures and, hence, both the orthosteric and the allosteric binding sites are the most accurate and most resembling those of the wild-type receptor (Figure 5A,B). Choosing a correct initial structure at this stage is essential since the completely different residues constitute the particular binding sites in different crystal structures.

Different conformations of the binding site in the crystal structures of $\mathrm{CB}_{1} \mathrm{R}$ are not only a result of different orthosteric ligands bound but, most importantly, they ensue from the presence or absence of the MPR and, particularly, of the C98-C107 disulfide bond (Figure 5B). Such claim is in agreement with experimental results showing that mutations within MPR weaken the binding of CP55,940 to the orthosteric binding site [35] while truncation of the whole $\mathrm{N}$-terminus at position 113 abolishes the binding completely. However, if only MPR is retained, the binding of the orthosteric ligands is maintained [29].

(A)

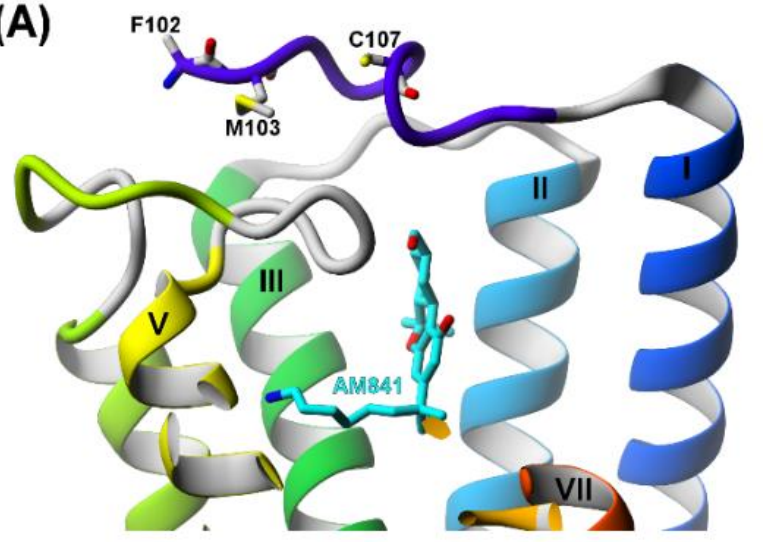

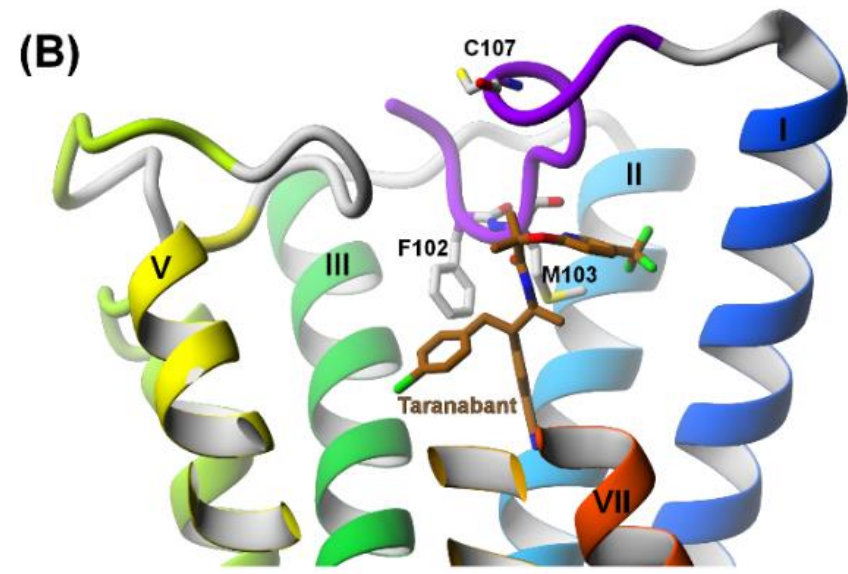

Figure 5. The influence of C98-C107 disulfide bond on the orthosteric binding of $\mathrm{CB}_{1} \mathrm{R}$. (A) PDB id:5XR8 structure with no disulfide bond; (B) PDB id:5U09 structure with disulfide bond present. In 5XR8 structure the residues F102 and M103 are outside of the orthosteric binding site; the ligand's position is shallower and there is much more space available in the orthosteric binding site so the ligand has more freedom and is in contact with many water molecules. In 5U09 structure the residues F102 and M103 are inside the orthosteric binding site; the ligand is positioned deeper, surrounded by hydrophobic residues and the binding is tighter.

We first elucidated the structure of MPR of $\mathrm{CB}_{1} \mathrm{R}$ (Figure 6) - see Section 3.1 of Methods for details. The structure of $\mathrm{CB}_{1} \mathrm{R}$ with MPR was used in our previous work [36] for investigating the entry pathway into the orthosteric binding site of the $\mathrm{CB}_{1}$ cannabinoid receptor. Next, the remaining residues $1-95$ of the N-terminal domain were added according to the sequence of a wild-type human $\mathrm{CB}_{1} \mathrm{R}$ and the conformational space of the newly added part was extensively explored using the Replica Exchange Molecular 
Dynamics with Solute Tempering [37] simulations. Details of this procedure are specified in Section 3.2 of Methods.

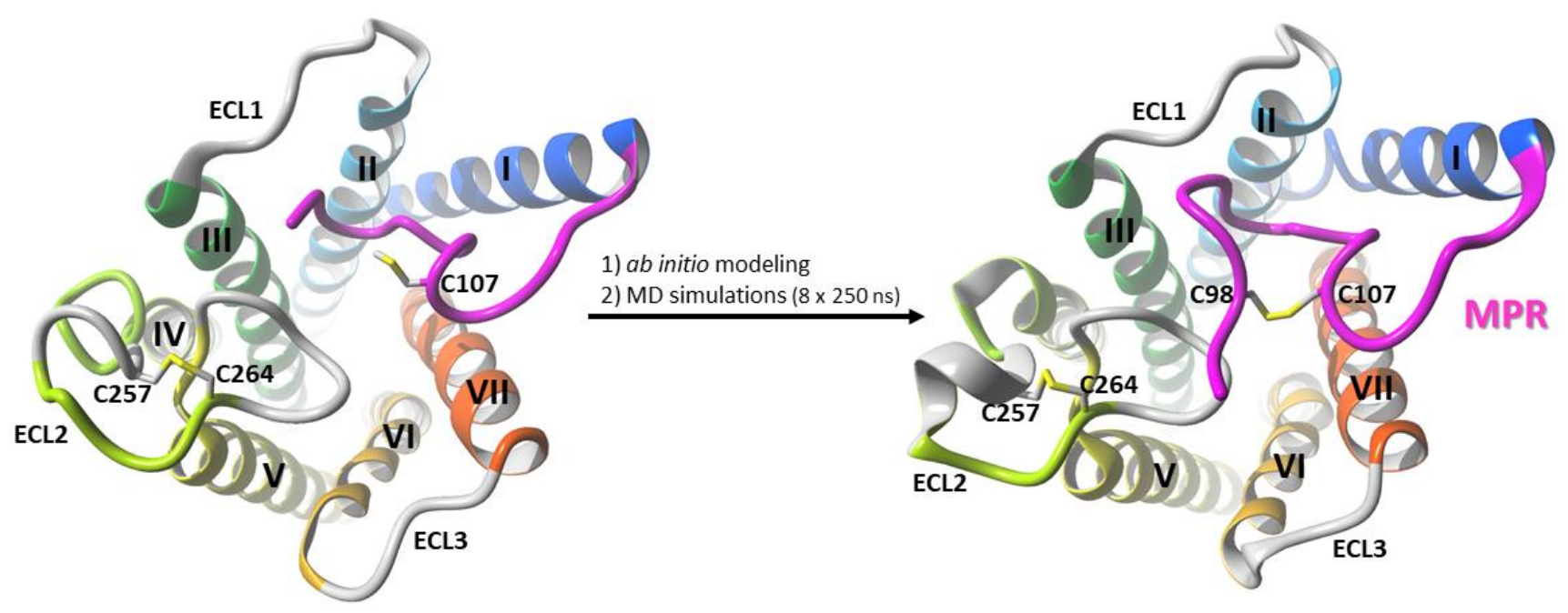

Figure 6. The modelling process of MPR of the $\mathrm{CB}_{1} \mathrm{R}$ N-terminal domain. Residues $96-99$ were added to the $\mathrm{CB}_{1} \mathrm{R}$ crystal structure (PDB ID: 5U09) and the C98-C107 disulfide bond was created. Regions 96-100 and 107-108 were then sampled in Rosetta. Eight structures of $\mathrm{CB}_{1} \mathrm{R}$ with MPR obtained from Rosetta were placed in POPC bilayer and simulated for $250 \mathrm{~ns}$.

\subsection{Structural Features of the $C B_{1} R N$-Terminal Domain}

The N-terminal helix 3 (NTH3, residues 80-94) is present in many structures obtained from REST2 simulations and, once it forms, it is very stable (Figure 7A).
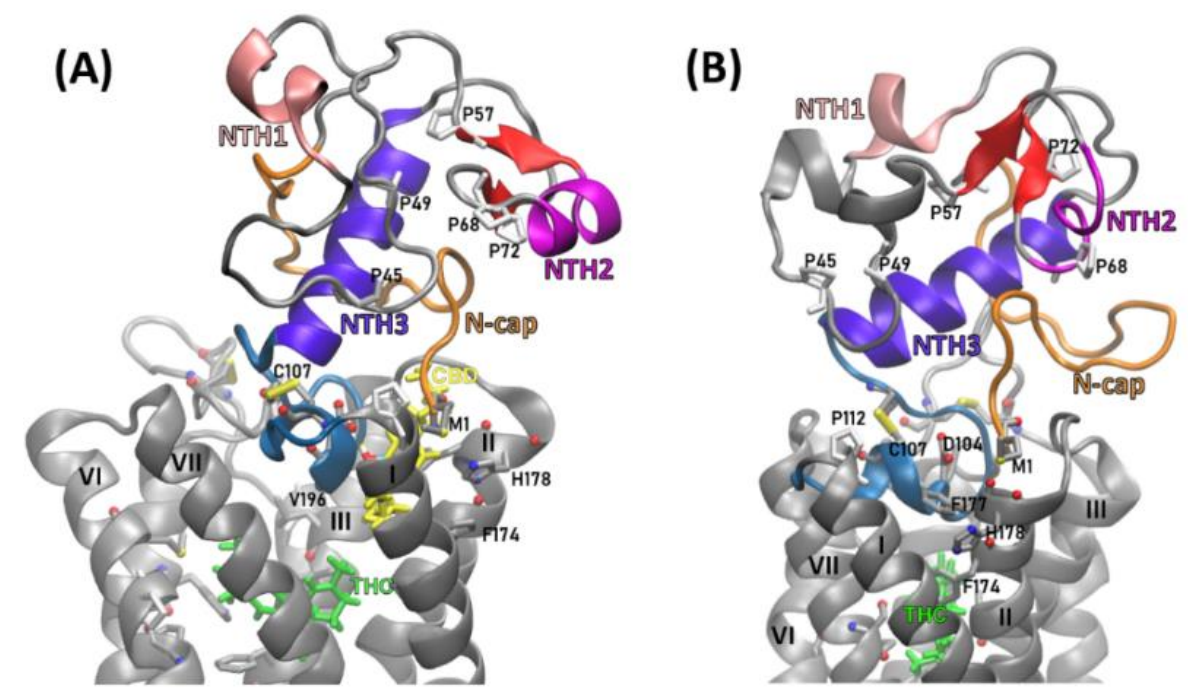

Figure 7. $\mathrm{CB}_{1}$ receptor $\mathrm{N}$-terminal domain conformation depends on $\mathrm{CBD}$ presence in the allosteric site. (A) $\mathrm{CB}_{1} \mathrm{R}$ with $\mathrm{CBD}$ and THC bound, (B) $\mathrm{CB}_{1} \mathrm{R}$ with THC bound only; cyan-MPR, violetN-terminal helix 3 (NTH3, residues 80-94), red-a parallel $\beta$-sheet (residues 58-60 and 72-74), magenta-N-terminal helix 2 (NTH2, residues 61-69), salmon-N-terminal helix 1 (NTH1, residues 30-35), orange- $\mathrm{N}$-terminal cap (residues 1-20). Proline residues are shown as white sticks.

Very likely this helix is a scaffold on which the rest of the N-terminus is arranged. The three glutamates, E91, E93, and E94, located in this helix, form ionic interactions with other parts of N-terminus (Figure 8A) constituting a hub essential for the N-terminal domain stability. For example, the 1-20 residue fragment, called by us the N-terminal cap (or simply 'N-cap') and located close to TM1 and TM2 extracellular ends, is relatively stable due to a salt-bridge K2-E93 (and also K2-D104 as well as K2-E106) (see the interactions on 
Figure 8A) that supports the conformation and position of this fragment. The residues M1 and $\mathrm{K} 2$ are within the allosteric site 1, constituting a barrier separating that site from the extracellular milieu. We have also observed a short parallel $\beta$-sheet forming repeatedly between the 58-60 and 72-74 strands while a very short helix formed occasionally within the 60-72 loop. The N-terminus contains five prolines: P45, P49, P57, P68 and P72, stacked together within a fragment of only 30 residues long. The stability of particular regions of N-terminus were estimated by RMSF calculations (Figure 9A,B) based on ten $1 \mu \mathrm{s}$ long $\mathrm{MD}$ simulations for $\mathrm{CB}_{1} \mathrm{R}$ complexes with and without $\mathrm{CBD}$. The additional six $\mathrm{MD}$ simulations $3 \mu \mathrm{s}$ long performed for corroboration of $\mathrm{N}$-terminus stability gave the structure of increased stability for the $\mathrm{CB}_{1}$ R complex with $\mathrm{THC}$ and without $\mathrm{CBD}$ (Figures $7 \mathrm{~B}$ and $9 \mathrm{C}$ ) where two additional short helices were formed but helix NTH2 was dissolved in one of three simulations.
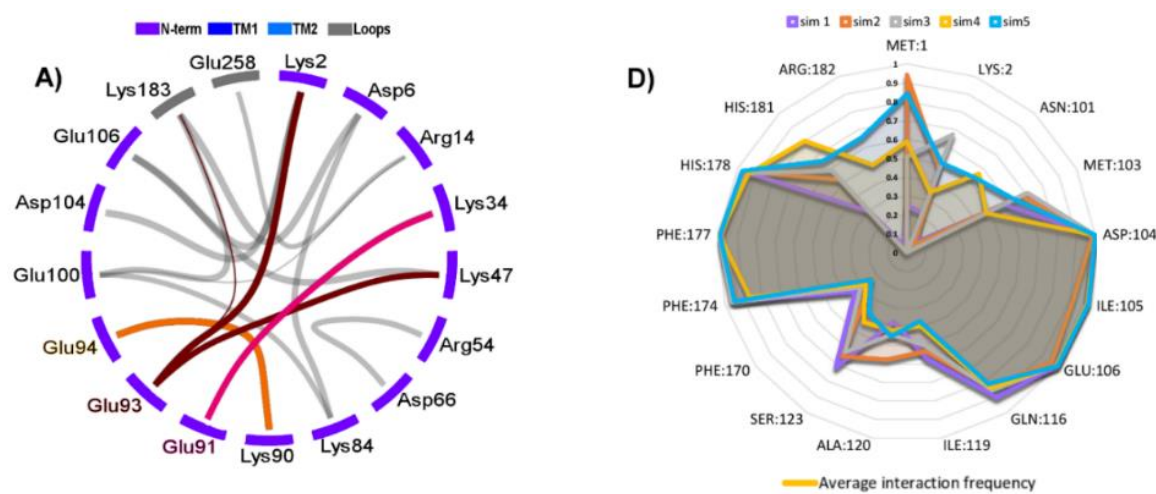

B)

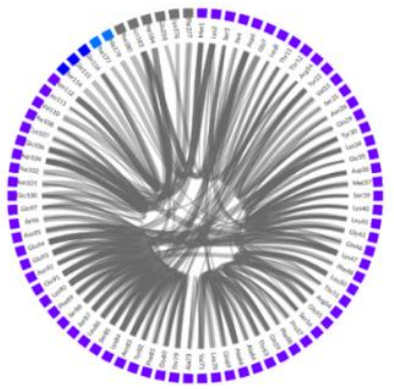

E)
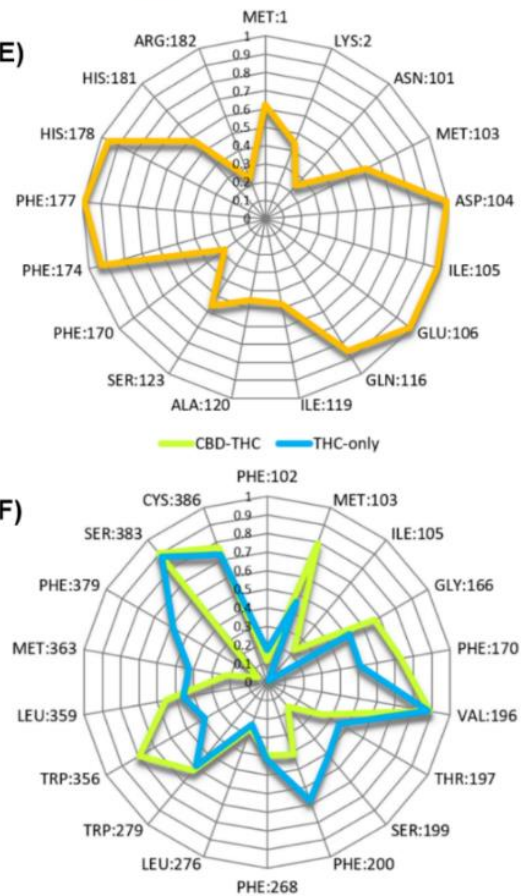

C

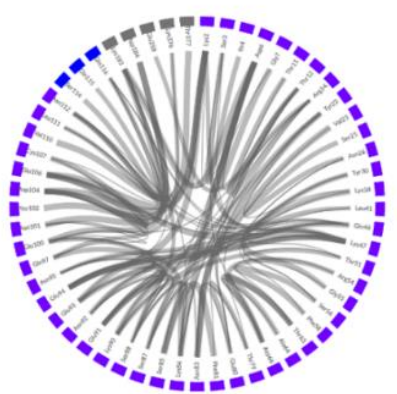

Figure 8. (A-C) Flare plots of crucial interactions responsible for stabilizing the N-terminal domain in THC-only complex). Line thickness is proportional to the frequency of the interaction; (A) Ionic interactions; highlighted are the interactions of E91 (pink), E93 (dark red) and E94 (orange), (B) Total hydrogen bonds of N-terminus; (C) The hydrogen bonds of N-terminus excluding backbonebackbone H-bonds; (D,E) Ligand-receptor dynamic interaction fingerprints in THC-CBD complex; (D) frequency fingerprints of interactions of residues with CBD for each simulation separately; (E) the average interaction frequencies of fingerprints from panel $\mathrm{D} ;(\mathbf{F})$ the average frequency fingerprints of interactions of residues with THC for simulations with THC-CBD (green) and with THC-only (blue) bound to the $C_{1} R$. 
(A)

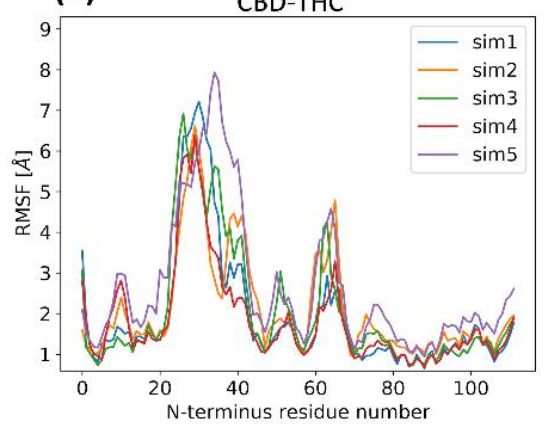

(B)

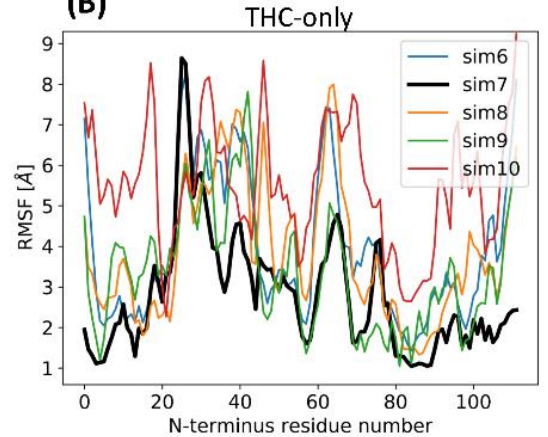

(C)

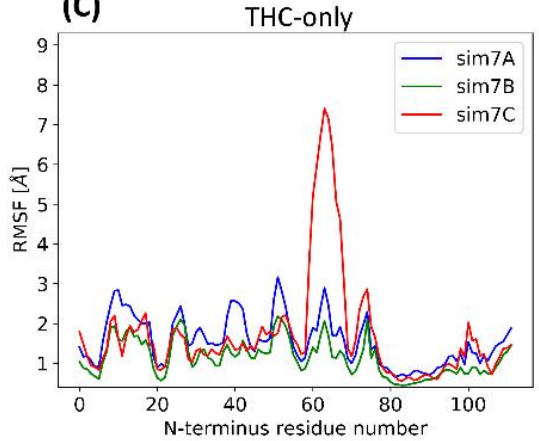

Figure 9. RMSF plots of $\mathrm{CB}_{1} \mathrm{R} N$-terminus (residues 1-113) measured during $1 \mu \mathrm{s}$ long $\mathrm{MD}$ simulations starting from the same receptor structure (Figure 4A) bound with (A) CBD and THC; (B) THC-only; (C) for simulations from N-terminus stability corroboration (Table 1). The last $1 \mu$ s from $3 \mu$ s MD simulations were taken for RMSF calculations.

As we had anticipated, the five prolines-containing fragment disrupts a formation of the larger secondary structure elements in that region and only transient structures can be formed. The whole $\mathrm{N}$-terminus is stabilized by internal salt bridges (Figure 8A) and a large number of internal hydrogen bonds but also forms a few interactions with the extracellular ends of TM1, TM2, and with the extracellular loops (Figure 8B,C).

\subsection{The Orthosteric and Allosteric Ligand Binding}

The analysis of ligand-receptor interaction patterns in the complex of $\mathrm{CB}_{1} \mathrm{R}$ with $\mathrm{CBD}$ and $\Delta^{9}$-THC (Figure 10) during MD simulations demonstrates that the pose of CBD is very stable when it is bound to the allosteric site 1 of a wild-type $\mathrm{CB}_{1}$ cannabinoid receptor with a whole $\mathrm{N}$-terminus which is indicated by existence of several ligand-receptor interactions with a frequency close to 1.0 for the residues D104, I105, E106 from MPR, Q116 ${ }^{1.32}$ from helix I, and residues F174 2.61 , F177 2.64 , H178 2.65 from helix II (Figure 8D,E). The important contribution to CBD binding comes also from the first residue of N-terminus, M1, which side chain partially protects CBD from the water environment.

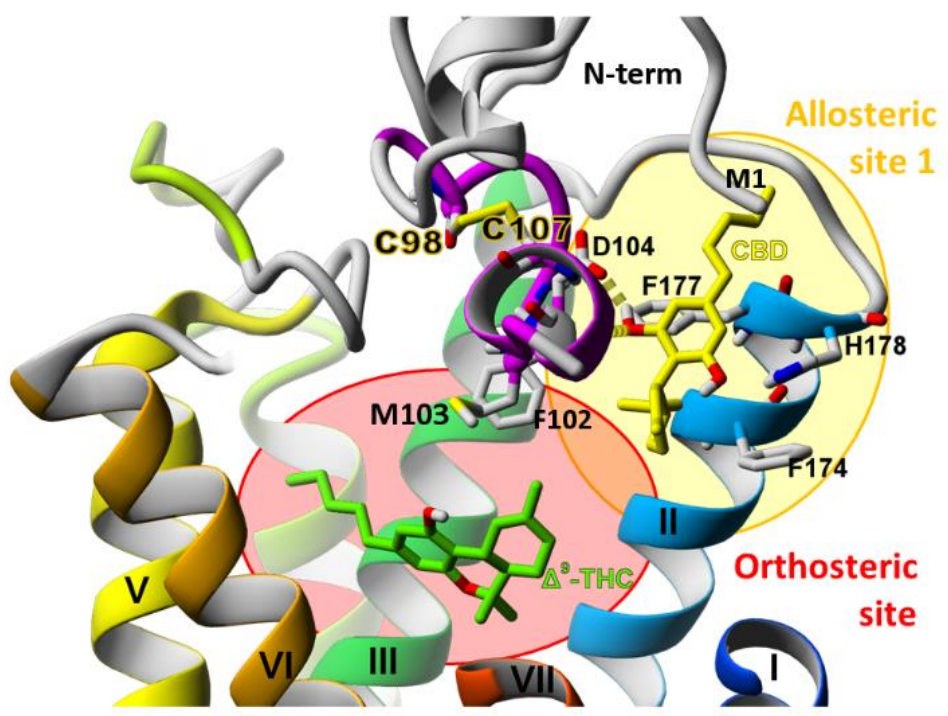

Figure 10. $\mathrm{CBD}$ and THC binding to $\mathrm{CB}_{1} \mathrm{R}$. $\mathrm{CBD}$ is bound to the allosteric site 1 while $\Delta^{9}-\mathrm{THC}$ is bound to the orthosteric site. The allosteric site is located very close to the orthosteric binding site and they partially overlap one another. The MPR (purple) constitutes a barrier between the orthosteric and the allosteric binding sites. CBD bound to the allosteric binding site forms a hydrogen bond with D104 and $\pi-\pi$ interactions with F177. 
The binding mode of THC in the orthosteric site is influenced by the presence of CBD in the allosteric site 1 . The residues for which the frequencies of interactions with THC are not changed are V196 $6^{3.32}, \mathrm{~T}_{197^{3.33}}$ of helix III, L276 ${ }^{5.40}$, W279 5.43 of helix V, L359 $9^{6.51}$ of helix VI, and S383 7.39 , C386 7.42 of helix VII (Figure 8F). CBD changes the average frequencies of interactions between THC and the receptor in such a way that some of them are increased and some decreased so the average binding pose of THC in the binding site is modified. The residues with increased frequencies are M103 of MPR, G166 ${ }^{2.53}$, F170 2.57 of helix II, and W356 $6^{6.48}$ of helix VI. The residues with decreased frequencies are S199 ${ }^{3.35}$, F200 3.36 of helix III, and M363 ${ }^{6.55}$ of helix VI (Figure 8 F). The residue W356 6.48 is a central residue of a transmission switch which is one of major molecular switches responsible for activation of GPCRs [38]. Molecular dynamics simulations for many GPCRs also suggest that the transmission switch is the first switch to be affected by the agonist binding $[39,40]$. Therefore, a modification of frequency of interactions between THC and the residues of the transmission switch (particularly F200 3.36 and W356 6.48 which are in tight proximity in $\mathrm{CB}_{1} \mathrm{R}$ ) by $\mathrm{CBD}$ presence in the allosteric site most likely corresponds to the negative allosteric modulator properties of CBD.

We have also compared the calculated ligand binding energies. The average THC-binding energy is lower (more negative, which means a more stable complex) by around $7.5 \%$ in THC-only simulations $(-53.9 \mathrm{kcal} / \mathrm{mol})$ than in CBD-THC simulations $(-50.1 \mathrm{kcal} / \mathrm{mol})$. Such observation is in agreement with experimental results indicating that allosteric modulation of $\mathrm{CB}_{1} \mathrm{R}$ by $\mathrm{CBD}$ decreases the binding affinity of $\mathrm{THC}$ and of the other orthosteric ligands [10].

In this paper we use for residues located in transmembrane helices the additional numbers in superscript which is the Ballesteros-Weinstein numbering [41] (extension of this scheme was proposed by Gloriam group [42]). This system was proposed for GPCRs to provide information about the relative positions of amino acids present in seven transmembrane helices. It contains two numbers separated by a dot: the first number indicates the number of the transmembrane helix (1-7) where the residue is located; the second number indicates a position of particular residue in relation to the most conserved residue in that helix, assigned number 50. Such numbers allow for direct comparison of residues among different GPCRs and especially in the most populated class A.

\subsection{Stability of the N-Terminal Domain}

The N-terminal domain of $\mathrm{CB}_{1}$ receptor was stable in all five CBD-THC simulations, however, it was significantly less stable in the THC-only simulations (Figure 9A,B) and underwent a rearrangement (Figure 9C). It should be noted that in the last stage of REST2 simulations we used a $C B D$ bound $C_{1} R$ and, therefore, we believe that the conformation of the $\mathrm{N}$-terminal domain accommodated the allosteric ligand. Although initially the N-terminus was less stable in the THC-only simulations when we continued one of the simulations ( $\operatorname{sim} 7$ in Figure $9 \mathrm{~B}$ ) the N-terminus structure converged to more stable conformation also containing NTH3 (residues 78-92), $\beta$-sheet between 58-60 and 72-74, NTH1 (residues 29-35) and a very stable 'N-cap' (residues 1-20) (Figure 7B). Therefore, we concluded that N-terminal domain is not only essential for stable binding of the allosteric ligands in 'allosteric site 1 ' but is also involved in allosteric modulation of the orthosteric binding. This hypothesis is supported by the mutagenesis studies of $C_{1} R$ [29]. When this conformation has been achieved, we confirmed its stability in three independent $3 \mu \mathrm{s}$ long MD simulations (Figure 9C). In one of the three simulations ( $\operatorname{sim} 7 \mathrm{C}$ ) the NTH2 helix together with the $\beta$-sheet (residues 58-74) were dissolved and therefore we observe high RMSF values for this region in simulation $7 C$. 


\subsection{The Effect of Truncation of the N-Terminus on the Energy of CBD Binding}

The $\mathrm{N}$-terminal domain of $\mathrm{CB}_{1} \mathrm{R}$ is essential for the stability of $\mathrm{CBD}$ in its allosteric binding site. In MD simulations of CBD-THC complex $(5 \times 1 \mu$ and more) with a whole $\mathrm{N}$-terminal domain none of the ligands exited its binding site (the average RMSD for each ligand was $\sim 2.5 \AA$ ). However, in all simulations of $\Delta-88$ (deletion of 1-88 residues) and $\Delta-98$ (deletion of $1-98$ residues) $\mathrm{CB}_{1} \mathrm{R}$ mutants (two simulations repeats for each mutant), the CBD binding mode was significantly distorted and the final pose was different for each of the four simulations (Figure 11). In two out of four cases (one simulation for $\Delta-88$ and one simulation for $\Delta-98$ ) CBD exited the allosteric site completely and within less than $500 \mathrm{~ns}$ it moved toward the orthosteric ligand binding channel between helices I and VII (this gate was described by us in our previous paper [36]) distorting the conformation of MPR significantly. In one of the two simulations for the $\Delta-8$ (deletion of $1-8$ residues) $C_{1} R$, CBD dissociated from the receptor completely after $350 \mathrm{~ns}$ and remained dissolved in the membrane for the rest of the simulation time.

The average RMSD of CBD was $12.5 \AA$ for $\Delta-8$ receptor, $5.7 \AA$ for $\Delta-88$, and $7.7 \AA$ for the $\Delta-98 \mathrm{CB}_{1} \mathrm{R}$ mutant and the average binding energies of $\mathrm{CBD}$ were $-63.29,-58.49$ and $-57.83 \mathrm{kcal} / \mathrm{mol}$, respectively, while for the wild-type receptor the binding energy was $-72.7 \mathrm{kcal} / \mathrm{mol}$ (Figure 12). The energies of CBD binding to mutants with a truncated $\mathrm{N}$-terminus $(\Delta-8, \Delta-88, \Delta-98)$ are significantly higher than for the wild-type receptor, suggesting that a whole $\mathrm{N}$-terminus is required for proper CBD binding.

(A)
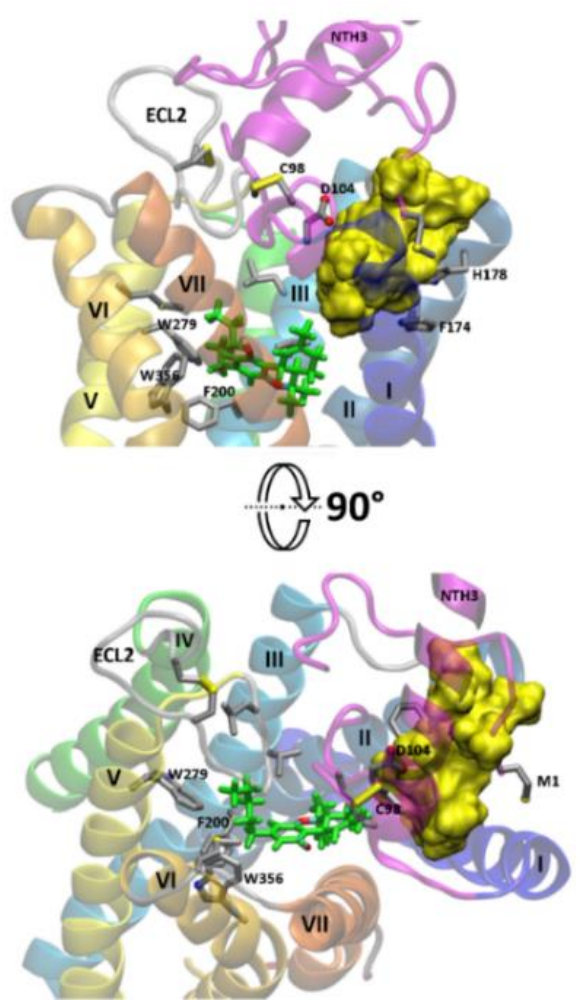

(B)
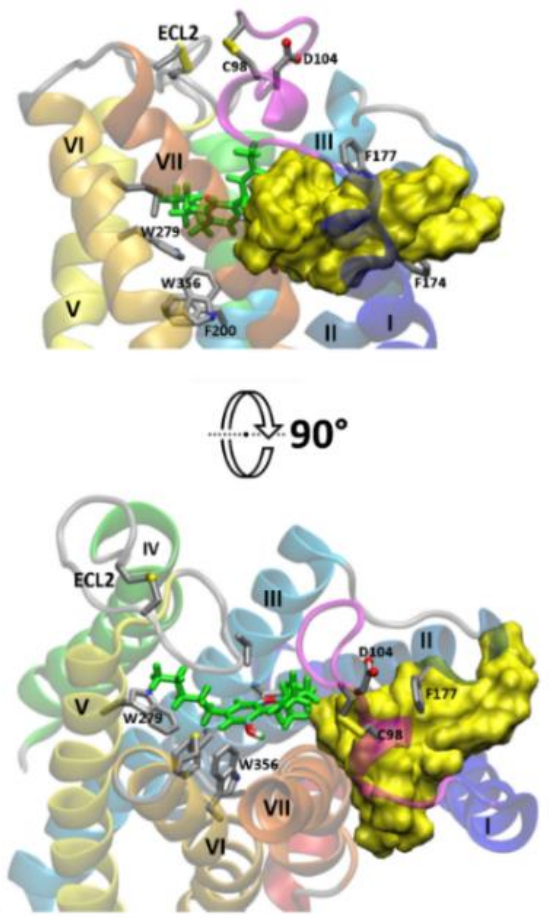

Figure 11. Comparison of CBD binding modes (yellow surface for all poses) obtained after $1 \mu \mathrm{s}$ of MD simulations for (A) WT $C_{1} R$ (with whole N-terminus), five CBD poses superimposed; and for (B) $\mathrm{CB}_{1} \mathrm{R}$ with truncated N-terminus-four $\mathrm{CBD}$ poses (two for $\Delta-88$ and two for $\Delta-98 \mathrm{CB}_{1} \mathrm{R}$ ). All poses were superimposed using receptor structure as a reference. Orthosteric agonist, THC, is shown in green. 
The average values of THC binding energy for $\Delta-8, \Delta-88$ and $\Delta-98$ mutants $(-51.1$, -52.0 and $-53.2 \mathrm{kcal} / \mathrm{mol}$, respectively) are lower than those for THC bound to a wild-type receptor with CBD in the allosteric site $(-50.1 \mathrm{kcal} / \mathrm{mol})$ and higher than those recorded for THC bound to the wild-type receptor without CBD $(-53.9 \mathrm{kcal} / \mathrm{mol})$ (Figure 12). It suggests that even if $C B D$ binds (at least to some extent) to $C_{1} R$ with truncated N-terminus its impact on THC binding, and perhaps also its NAM activity, will be significantly reduced. Our results indicate that the larger fragment of the N-terminus is deleted the lower is the influence of CBD binding on the binding energy of THC (Figure 12).
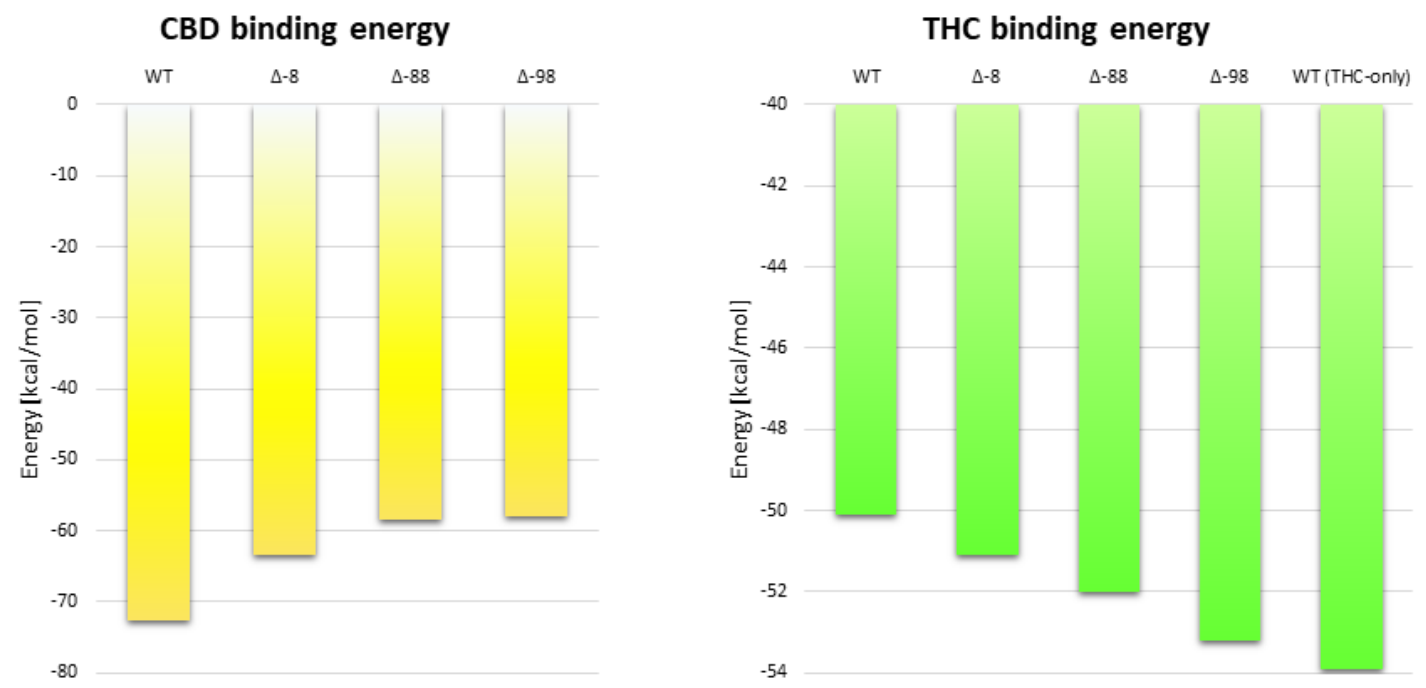

Figure 12. Comparison of ligand binding energies for different mutants of $C B_{1} R$ with truncated $N$-terminus and a wild-type receptor for CBD-THC complex. The energies of CBD binding to the allosteric site (left) are shown in yellow while the energies of THC binding to the orthosteric site (right) are shown in green. All reported binding energies are the average values obtained from several ( 5 for WT, and 2 for mutants) $1 \mu$ s long MD simulations and they were performed for CBD-THC complex (except for the last rectangle). $\Delta-8$ (deletion of $1-8$ residues), $\Delta-88$ (deletion of $1-88$ residues) and $\Delta-98$ (deletion of 1-98 residues).

\subsection{The Dynamic Contact Networks}

The dynamic contact networks for CBD-THC and THC-only simulations are different. The most significant changes in frequencies of certain interactions in MD simulations are observed for hydrogen bonds, water mediated hydrogen bonds and van der Waals interactions networks (Figure 13). The interaction frequency and the interaction frequency difference $\left(\Delta_{\text {freq }}\right)$ is defined in Section 3.5 of Methods.

Comparison of the contact networks of CBD-THC and THC-only MD simulations has revealed that several residues from the N-terminal domain (such as E91, E94, N95, I96, and C107) participate in the allosteric interaction network essential for the communication between the allosteric and orthosteric binding sites. Importance of disulfide bond C98-C107 for the allosteric-orthosteric coupling was strengthened since one of its residues, C107, formed a hydrogen bond with residue N95 in all MD simulations of CBD-THC complex while this bond was broken in all MD simulations of THC-only complex (Figure 13A,D). We have also found that the residues V110 ${ }^{\mathrm{MPR}}, \mathrm{K} 373^{7.29}$ and $\mathrm{T} 377^{7.33}$ constitute a switch that is involved in the allosteric modulation of $\mathrm{CB}_{1} \mathrm{R}$ by $\mathrm{CBD}$. In the presence of $\mathrm{CBD}$ a hydrogen bond V110-T377.33 is predominantly formed (Figure 14A) while in absence of CBD a hydrogen bond K373 ${ }^{7.29}-\mathrm{T}_{377^{7.33}}$ is formed much more often (Figure 14B). For those interactions we observed one of the highest average frequency differences between CBD-THC and THC-only systems, 0.78 and -0.74 , for V110-T377 ${ }^{7.33}$ and K373 ${ }^{7.29}-$ T377 $^{7.33}$, respectively (H-bonds in Figure 13A,D). 

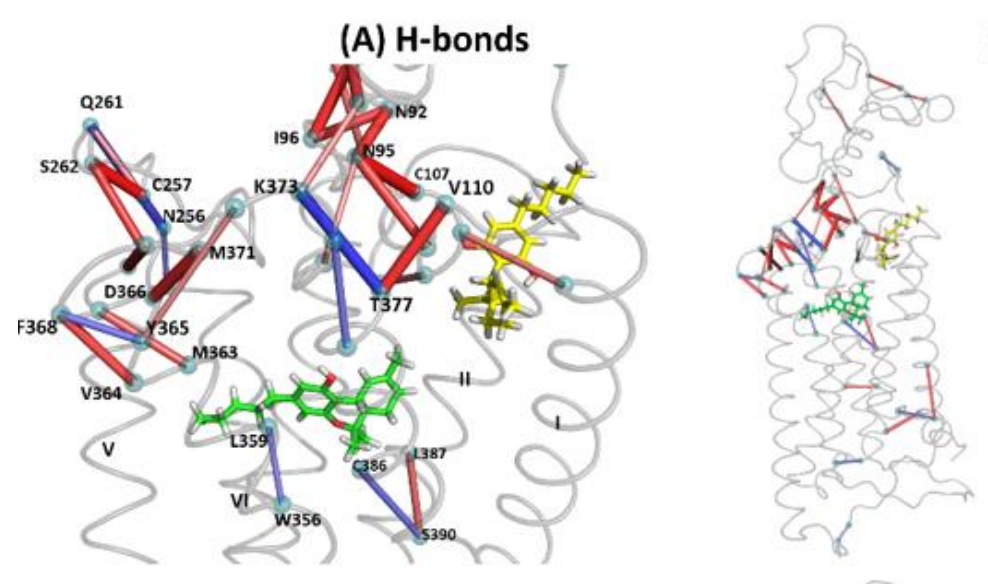

(B) Water mediated $\mathrm{H}$-bonds

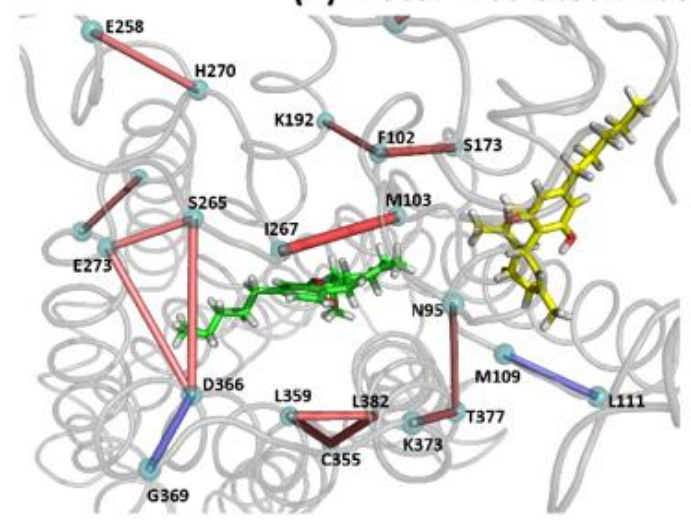

(C) VdW interactions

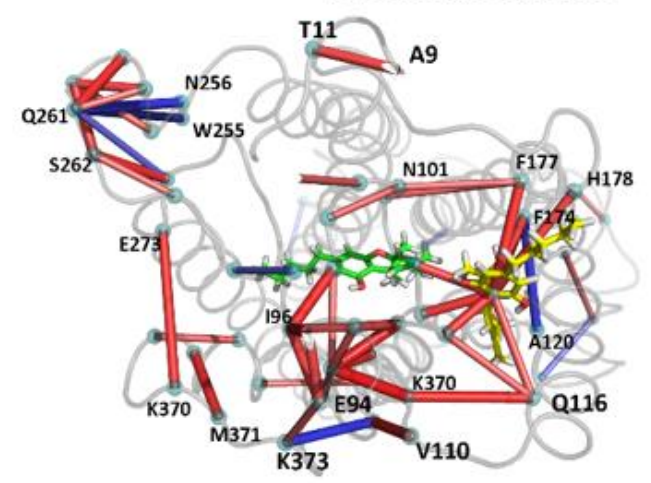

(D)
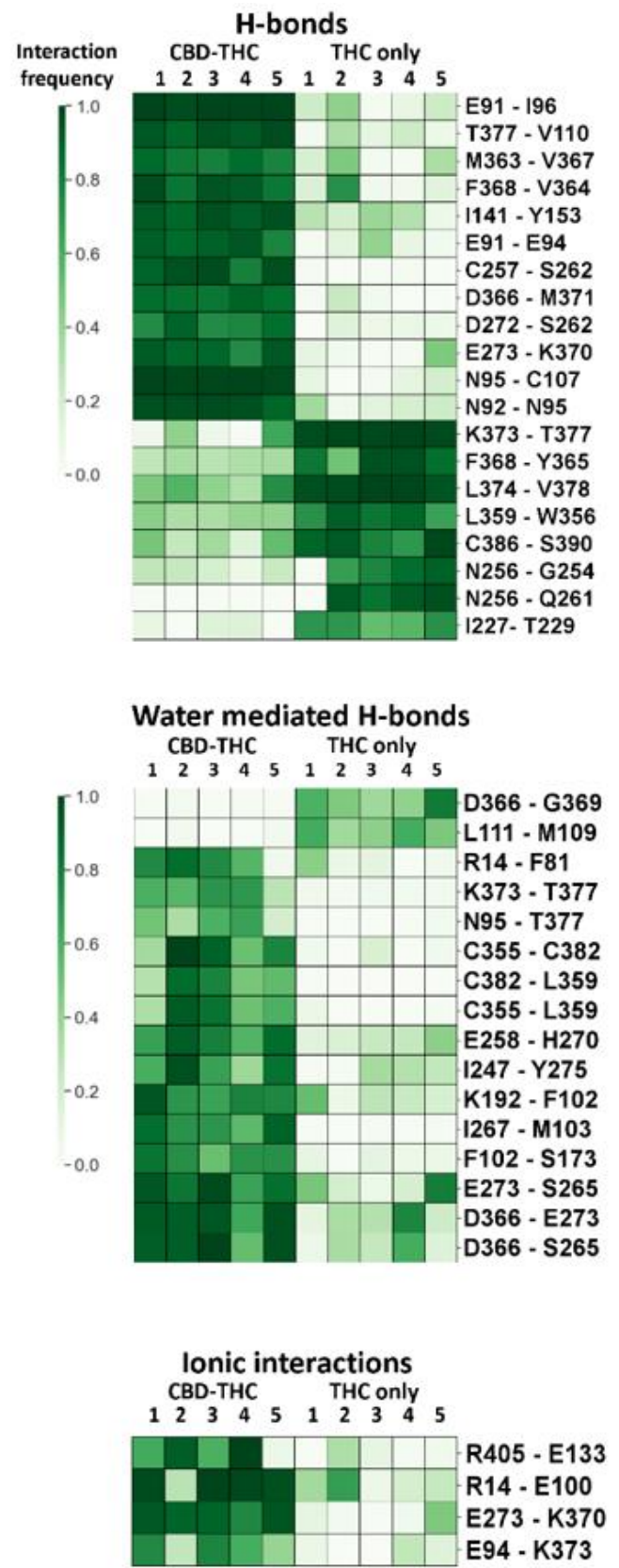

Figure 13. Differences in contact frequencies between CBD-THC and THC-only complexes; (A) Hydrogen bonds-both side views; (B) Water mediated hydrogen bonds—the extracellular and side view; (C) van der Waals interactions-the extracellular and side view. Red sticks represent contacts occurring more frequently for CBD-THC simulations, and blue ones are the contacts occurring more frequently in THC-only simulations. The thickness of a stick as well as a color intensity correspond to the average frequency difference between those two populations of trajectories; (D) The interaction frequency fingerprints for CBD-THC and THC-only complexes ( 5 simulations for each complex). Presented interactions are very likely participating in the allosteric-orthosteric communication network since their frequencies differ significantly between simulations with and without an allosteric ligand (CBD). Among all possible interactions only the hydrogen bonds, water mediated hydrogen bonds, and ionic interactions are shown. 

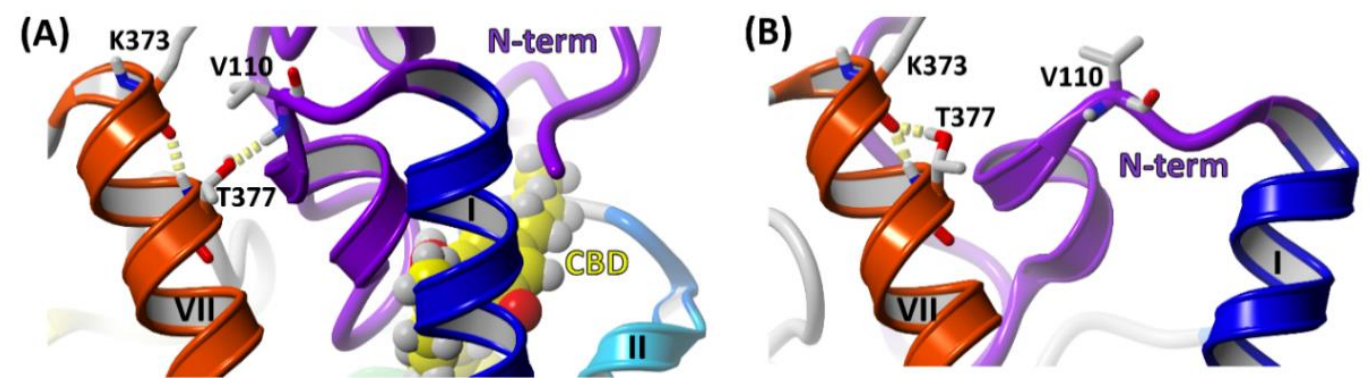

Figure 14. The molecular switch formed by residues $\mathrm{V} 110^{\mathrm{MPR}}-\mathrm{T} 377^{7.33}-\mathrm{K} 373^{7.29}$ as an important element of the allosteric modulation. (A) In CBD-THC simulations the switch exists predominantly in the configuration where a hydrogen bond V110 ${ }^{\mathrm{MPR}}-\mathrm{T}_{377^{7.33}}$ (side chain-main chain) is formed; (B) in THC-only simulations the hydrogen bond of side chain of T377 7.33 is much more often formed within the same helix VII to K373 ${ }^{7.29}$ (side chain-main chain).

The residue M103 ${ }^{\mathrm{MPR}}$, which is located between the allosteric and orthosteric sites (Figure 10), interacts both with CBD in the allosteric site and with THC in the orthosteric site, and the frequencies of these interactions are high in CBD-THC complex: 0.61 for interaction with CBD (Figure 8E), and 0.80 for interaction with THC (Figure 8F). We conclude that $\mathrm{M} 103^{\mathrm{MPR}}$ could be relevant for the allosteric-orthosteric communication since the frequency of the M103-THC interaction is highly dependent on the presence or absence of CBD in the allosteric site, 0.80 in CBD-THC complex, and 0.46 in THC-only complex (Figure 8F). The residue $\mathrm{I} 105^{\mathrm{MPR}}$ is also located between the allosteric and orthosteric sites, however, the frequencies for I105-THC interaction are very low (Figure $8 \mathrm{~F}$ ), and therefore we cannot say if this residue participates in the allosteric-orthosteric coupling.

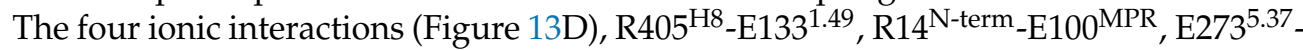
$\mathrm{K} 370^{\mathrm{ECL} 3}$, and $\mathrm{E} 94^{\mathrm{MPR}}-\mathrm{K} 373^{7.29}$ were found significantly more often in the CBD-THC complex than in THC-only, with the average frequency difference $\left(\Delta_{\text {freq }}\right): 0.46,0.53,0.54$ and 0.75 , respectively. Perhaps, the mutagenesis studies could elucidate the influence of the above residues on the allosteric-orthosteric coupling. The extracellular loop 2 (ECL2), and particularly the residues N256, C257, Q261, S262, also participate in the allostericorthosteric coupling mechanism especially that in some THC-only simulations we observed the interactions between N-terminal domain and ECL2 loop. Interestingly, not only the Nterminus residues but also some residues from cytoplasmic part of the receptor (Figure 7C) are involved in the allosteric modulation and can influence the cellular response.

\section{Methods}

\subsection{Modeling of the Membrane-Proximal Region (MPR) of $C B_{1} R$}

The crystal structure of $\mathrm{CB}_{1} \mathrm{R}$ (PDB id: 5U09) was converted to the wild-type (T210A mutation reversed), the residues 96-99 were added using YASARA v.19.1.27 program [43] and the regions 96-100 and 107-108 were then sampled using the Rosetta loopmodel protocol [44] with a condition that the C98-C107 disulfide bond must be maintained. From the 500 models obtained eight were selected based on Rosetta total score. Those eight selected structures were placed into a hydrated POPC bilayer and $250 \mathrm{~ns}$ of a full atom MD simulation was performed in AMBER v.18 program [45] for each of them. In five out of eight simulations the 96-112 fragment converged to a similar stable structure and the one with the lowest nonbonded energy of MPR was selected for further studies.

\subsection{Building and Elucidating the Structure of Whole N-Terminus}

The remaining 95 residues of the N-terminal domain (residues 1-95) were added from the sequence of a wild-type human $\mathrm{CB}_{1} \mathrm{R}$ obtained from the Uniprot database [46]. The BuildLoop command from YASARA [47] was used to build the initial model of Nterminus and its conformation was sampled using the Rosetta loopmodel protocol [44]. The selected structures from the clustered Rosetta solutions were used for exploration of 
the conformational space of the $\mathrm{CB}_{1} \mathrm{R} N$-terminal domain employing the Replica Exchange Molecular Dynamics with Solute Tempering (REST2 [37]) algorithm working within NAMD 2.13 platform [48] using CHARMM36m force field [49]. The all-atom REST2 simulations were performed for the whole $\mathrm{CB}_{1}$ cannabinoid receptor (residues 1-412) with the $\mathrm{N}$ terminus (residues 1-98, unless specified otherwise) selected as a hot region (Figure 15).

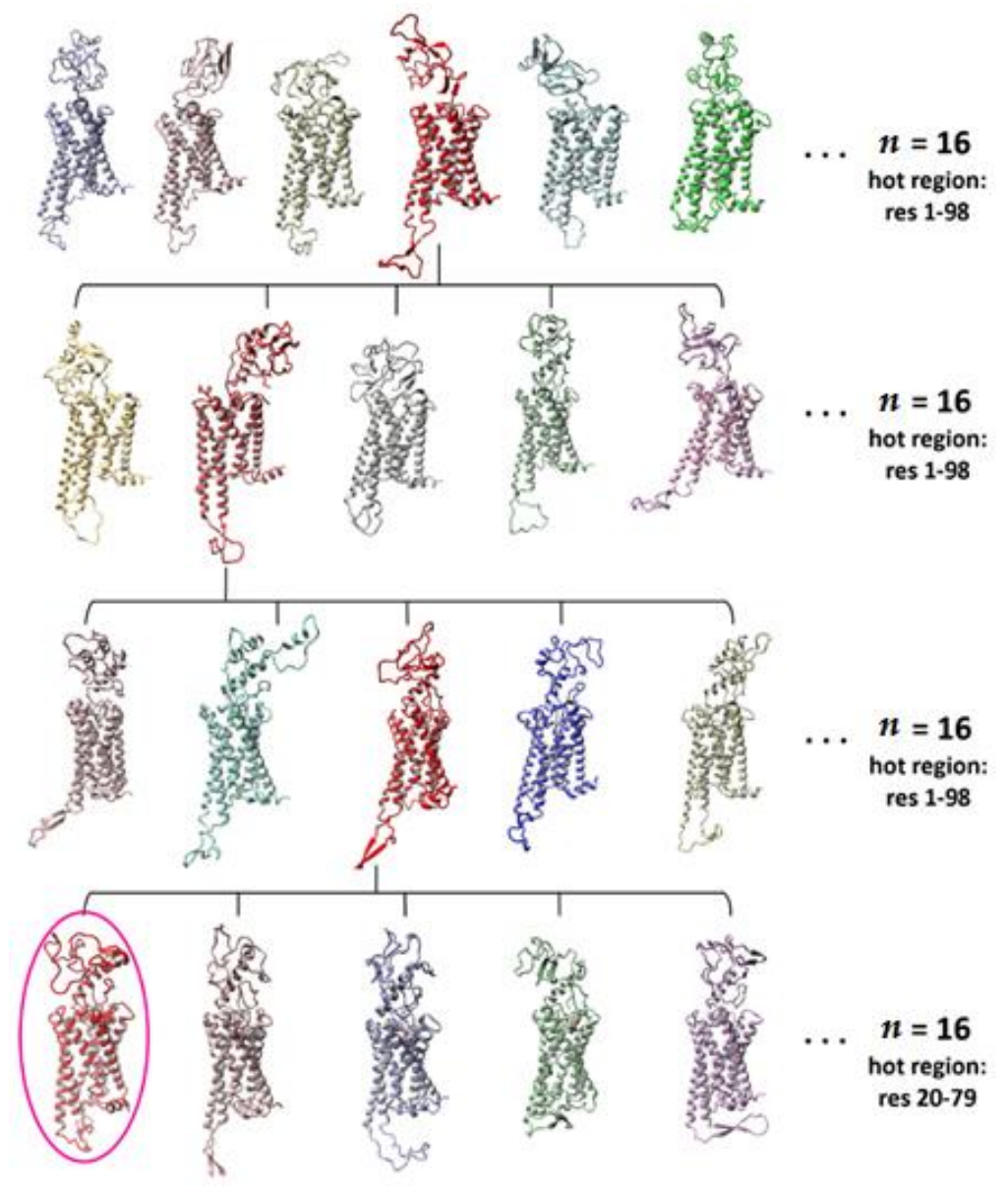

Figure 15. Sample structures of $\mathrm{CB}_{1}$ receptor with complete $\mathrm{N}$-terminal domain at each selection step of the REST2 MD simulations. The span of hot region of N-terminal domain and number of replicas in each step are specified on the right.

The receptor was immersed in a hydrated 1-palmitoyl-2-oleoyl-sn-glycero-3-phosphocholine (POPC) bilayer enriched by $20 \%(n / n)$ cholesterol. The whole system was generated in CHARMM-GUI webserver [50] which was used for system preparation and input files generation. The standard system contained $~ 70,000$ atoms and $~ 130$ lipid molecules (cholesterol + POPC) including $\mathrm{Na}^{+}$and $\mathrm{Cl}^{-}$counter ions in total ion concentration $0.15 \mathrm{M}$ for system charge neutrality. The average dimensions of a periodic cell were $74 \AA \times 74 \AA \times 140 \AA$. The TIP3P water model, parametrized to use with CHARMM force fields, was employed. Each REST2 simulation was run in 16 replicas, all starting from the same initial structure. All simulations were performed using the $2 \mathrm{fs}$ time step and all bond lengths to hydrogen atoms were constrained using SHAKE [51] algorithm allowing to use a longer time step than $1 \mathrm{fs}$. The actual temperature of the system was $300 \mathrm{~K}$, while the interactions for the hot region were scaled for temperatures 300-600 K. Temperature exchange attempts were performed every 100 steps (200 fs). The electrostatic interactions cutoff was set to $12 \AA$ with 10-12 A switching function, while the cutoff for pairlist generation was set to $13.5 \AA$. Long-range electrostatic interactions were computed using the Particle Mesh Ewald [52] summation scheme with PMEGridSpacing parameter set to 1.0. In the above simulations the group pressure and the Langevin dynamics were used. The best structures of $C_{1} R$ 
were elucidated from REST2 simulations based on their lowest nonbonded energies and low local RMSF values of the N-terminus during the simulations. For the last step of REST2 simulation the residues 20-79 instead of 1-98 were selected as the hot region due to their high RMSF values and CBD was docked to the allosteric site (Figure 15).

\subsection{Ligand Docking}

The most stable structure of $\mathrm{CB}_{1} \mathrm{R}$ with the whole N-terminus obtained from REST2 simulations and selected based on RMSF and energy analysis, was used for ligand docking. CBD was redocked to the allosteric site 1 [31] (positioned between the extracellular ends of TM1 and TM2, defined by residues: I105, E106, C107) after the last step of REST2 set of simulations, while $\Delta^{9}$-THC was docked to the orthosteric binding site (defined by residues: I119, F174, L193, W356, F381, S383, M384) [34]. To perform docking of flexible ligands into protein binding sites we used Genetic Optimization for Ligand Docking (GOLD) v.5.7.3 [53]. Ligand poses were verified for stability by MD simulations. Only one stable pose was identified for CBD and it was stable for $>1 \mu$ s of total MD simulation (in 5 simulation repeats). For each of the two ligand-receptor complexes (1st complex of $\mathrm{CB}_{1} \mathrm{R}$ with $\mathrm{CBD}$ and $\mathrm{THC}$, and 2 nd complex of $\mathrm{CB}_{1} \mathrm{R}$ with THC only) five independent all-atom and unbiased MD simulations were performed $(2 \times 5 \times 1 \mu \mathrm{s})$.

\subsection{All-Atom $M D$ Simulations of $C B_{1} R$ Receptor with Ligands}

Standard molecular dynamics simulations of the $\mathrm{CB}_{1}$ cannabinoid receptor with appropriate ligands immersed in a hydrated POPC bilayer (enriched by $20 \%(n / n)$ cholesterol), were performed in AMBER18 using CHARMM36m force field [49]. All systems were generated in CHARMM-GUI service [50] and the receptor orientation in the membrane was set according to the OPM database [54]. The standard system contained $\sim 70,000$ atoms, 127 lipid molecules (cholesterol + POPC), $43 \mathrm{Na}^{+}$cations and $50 \mathrm{Cl}^{-}$anions (total ion concentration was $0.15 \mathrm{M}$ ). The average dimensions of a periodic cell were $74 \AA \times 74 \AA \times 140 \AA$. TIP3P water model parametrized to use with CHARMM force fields, was employed. Before each MD simulation the system was submitted to a restrained energy minimization (5000 cycles). The first 2500 minimization cycles were performed with a steepest-descent method and after that conjugate gradient was switched on. Next, a six-step equilibration was performed (375 ps total) at a constant pressure and temperature (NPT ensemble; 310 K, 1 bar). During equilibration the restraints were released gradually, until the last step (last 100 ps of equilibration), in which no restraints were used. In the production simulations (as well as in the last three equilibration steps) all bond lengths to hydrogen atoms were constrained using SHAKE algorithm [51,55], which allowed us to use a longer time step ( $2 \mathrm{fs}$ instead of $1 \mathrm{fs}$ ). Van der Waals and short-range electrostatic interactions cutoff $=12 \AA$, with 10-12 $\AA$ switching function, were used. Long-range electrostatic interactions were computed using the Particle Mesh Ewald [52] summation scheme.

The MD simulations of $1 \mu$ s lengths each were performed for the CB1 receptor with the complete N-terminus (model obtained from REST2 simulations) and the following ligands bound: (1) five simulations for $\Delta^{9}$-THC in the orthosteric binding site and CBD in the allosteric binding site, and (2) five simulations for $\Delta^{9}-\mathrm{THC}$ in the orthosteric binding site and without the allosteric ligand.

\subsection{Contact Network Analysis}

The Pytraj tool (https:/ / github.com/Amber-MD/pytraj (accessed on 6 March 2021)), a Python front-end of the popular cpptraj package [56], was used for trajectory processing. GetContacts software (https:/ / getcontacts.github.io/ (accessed on 6 March 2021)) was used for analysis of the contact network within the receptor, and for calculation of the ligandreceptor and receptor-receptor interaction frequencies in each MD trajectory. The Flare plots (https:/ / gpcrviz.github.io/flareplot/ (accessed on 6 March 2021)) were used to visualize the residue-residue interactions. Based on the trajectory from the MD simulations using get_dynamic_contacts.py tool, we created a residue contact list for the MD simulation 
range $100 \mathrm{~ns}-1 \mu \mathrm{s}$. Since all MD simulations started from the same structure of $\mathrm{CB}_{1} \mathrm{R}$ selected using the REST2 simulations, by skipping the first $100 \mathrm{~ns}$ we hoped to highlight the differences between the contact networks of receptors bound with a different set of ligands. Using get_contact_frequencies.py module we calculated contact frequencies for all interactions in each trajectory. The interaction frequency (Freq) ranges between 0 and 1: Freq $=1.0$ means that a particular interaction is present in every single trajectory frame, Freq $=0.5$ means the interaction is found only in $50 \%$ of the frames, while Freq $=0$ means the interaction does not occur in the analyzed trajectory. By comparing contact frequencies between two sets of trajectories (1st for $\mathrm{CB}_{1} \mathrm{R}$ with $\mathrm{CBD}$ and $\mathrm{THC}$, and 2 nd for $\mathrm{CB}_{1} \mathrm{R}$ with THC-only; 5 trajectories for each set of ligands) we constructed the interaction fingerprints. The cutoff for residue-residue interaction fingerprint was set to 0.5 (which means that a particular interaction is considered only if its frequency is $\geq 0.5$ in at least one of the trajectories) and for ligand-protein interaction fingerprints the cutoff was set to 0.3 .

Based on the analysis of numerical interaction fingerprints, we elucidated the interactions that are highly dependent on the presence or absence of CBD in the THC bound $\mathrm{CB}_{1}$ receptor. We performed a statistical analysis of the frequency distributions in two sets of trajectories and calculated the difference between average frequencies of a particular contact $\left(\Delta_{\text {freq }}\right.$, described by Equation (1)) in order to identify those contacts for which the frequency differed significantly between $\mathrm{CBD}-\mathrm{THC}$ and $\mathrm{THC}$-only $\mathrm{CB}_{1}$ receptor complexes. The Student's t-test was performed to verify whether the distributions of frequencies are different between two sets of trajectories comparing the average frequencies of each contact between those sets. If the frequency distributions are classified as different $(p$ value $<0.05)$ and the absolute value of the average frequency difference between the two populations of trajectories is higher than $0.4\left(\left|\Delta_{\text {freq }}\right|>0.4\right)$ for a particular contact, then the contact is kept as a potentially meaningful for the allosteric-orthosteric coupling mechanism. We visualized the average frequency differences for these meaningful interactions as red and blue sticks linking the interacting residues (Figure 13A-C).

$$
\Delta_{\text {freq }}=\overline{\text { Freq }_{(C B D+T H C)}}-\overline{\text { Freq }(T H C-\text { only })}
$$

\subsection{Mutational Study of N-Terminus on CBD Binding to the CB1 Receptor}

In order to verify if the presence of the $\mathrm{N}$-terminus is relevant for the allosteric binding of $C B D$ to the $C_{1}$ receptor, we performed all-atom MD simulations of $1 \mu$ s lengths for $\mathrm{CB}_{1} \mathrm{R}$ with $\mathrm{N}$-terminus truncated at various sites $(\Delta-8, \Delta-88, \Delta-98)$ bound to $\mathrm{CBD}$ (in the allosteric site 1) and THC (in the orthosteric site). Then, we compared the binding energies and the RMSD values of ligands in all $C_{1} R$ mutants with those values for ligand-bound wild-type receptor.

\section{Conclusions}

This is the first reported attempt of model the whole $\mathrm{N}$-terminal domain of $\mathrm{CB}_{1} \mathrm{R}$ concluded by obtaining a relatively stable structure of this domain maintained throughout multiple MD simulations. Although for some parts of the N-terminus the RMSF values are in range 7-8 $\AA$ it also contains very stable regions with RMSF in range $1-3 \AA$. Besides, it cannot be ruled out that some regions of the N-terminus are flexible by nature and their conformation is highly sensitive to the allosteric ligand bound to allosteric site. This might explain why no experimental structure of the $C_{1} \mathrm{R} N$-terminal domain has been determined so far. We observed that the particular conformation of $\mathrm{N}$-terminus obtained for CBD-THC-CB $\mathrm{C}_{1}$ complex was stable in all five simulations but it underwent a rearrangement in all five simulations of the $T H C$-only- $C B_{1} R$ complex. Continuation of one of these MD simulations ( $4 \mu$ s in total) led to a different stable conformation of the $\mathrm{N}$-terminus of the THC-only- $\mathrm{CB}_{1} \mathrm{R}$ complex.

We found that CBD bound to the allosteric site is stable only when the whole Nterminus of $\mathrm{CB}_{1} \mathrm{R}$ is present. In the case of $\Delta-8, \Delta-88$ and $\Delta-98$ mutants (deletion of $1-8$ residues, 1-88 residues, and 1-98 residues, respectively), the binding energy of CBD is 
significantly higher, indicating a less stable complex, and the ligand dissociates from the binding site in 50\% of cases. By analyzing of MD simulations of CBD-THC and THConly complexes, and comparing the residue contact networks within those systems, we elucidated the major interactions responsible for the allosteric modulation of $\mathrm{CB}_{1} \mathrm{R}$ by $\mathrm{CBD}$ : (i) the N-terminus residues with a possible molecular switch involving residues V110 $\mathrm{MPR}$, $\mathrm{K} 33^{7.29}$ and $\mathrm{T} 377^{7.33}$ with alternating hydrogen bonds between them, and (ii) the residues from the extracellular loop 2 (ECL2).

The results of MD simulations indicate that the $\mathrm{N}$-terminal domain of $\mathrm{CB}_{1} \mathrm{R}$ plays a critical role not only in the binding of CBD (and perhaps the other allosteric modulators) but also for the allosteric-orthosteric coupling mechanism. Better understanding of the role of the $\mathrm{CB}_{1} \mathrm{R} \mathrm{N}$-terminal domain, including elucidation of the allosteric-orthosteric interaction network, is extremely important for understanding the allosteric modulation mechanism in $\mathrm{CB}_{1} \mathrm{R}$ and can be potentially useful in a design of novel allosteric modulators. All of the above findings on the role of $\mathrm{N}$-terminal domain need the experimental confirmation but they are also useful on their own for molecular modelers and also for drug discovery and design purposes.

Author Contributions: Conceptualization, J.J. and S.F.; methodology, J.J.; software, P.P.; validation, J.J., R.A. and R.P.; formal analysis, S.F.; investigation, J.J. and R.A.; resources, P.P.; data curation, J.J. and U.O.; writing—original draft preparation, J.J.; writing—review and editing, J.J. and S.F.; visualization, J.J. and U.O.; supervision, S.F.; funding acquisition, J.J. All authors have read and agreed to the published version of the manuscript.

Funding: This research was funded by National Science Centre, Poland, grant PRELUDIUM 2016/23/N/NZ1/02960 for J.J. Part of computational work has been done at the Interdisciplinary Centre for Mathematical and Computational Modelling in Warsaw (grant no. G07-13 and GA71-27).

Institutional Review Board Statement: Not applicable.

Informed Consent Statement: Not applicable.

Data Availability Statement: The data supporting reported results are deposited at Faculty of Chemistry, University of Warsaw, and are available upon request.

Acknowledgments: We would like to thank Sunhwan Jo for consultations, priceless advice and a huge help with running REST2 simulations, Jana Selent and Tomasz Stẹpniewski for recommending GetContacts software used for contact network analysis, the whole CHARMM-GUI team for creating a great open access web service for MD input generation, Szymon Niewieczerzał for scientific consultations and Przemysław Miszta for help with software installation. The European COST Action CA18133 (ERNEST) is acknowledged for providing biannual forum for exchange ideas on GPCR research and for supporting mutual visits.

Conflicts of Interest: The authors declare no conflict of interest. The funders had no role in the design of the study; in the collection, analyses, or interpretation of data; in the writing of the manuscript, or in the decision to publish the results.

Sample Availability: The structures and trajectories of CBD-THC- $\mathrm{CB}_{1} \mathrm{R}$ and THC-only-CB $\mathrm{C}_{1} \mathrm{R}$ complexes are available from the authors.

\author{
Abbreviations \\ 2-AG 2-arachidonylogycerol \\ AEA $\quad \mathrm{N}$-arachidonoylethanolamine (anandamide) \\ $\mathrm{CB}_{1} \mathrm{R} \quad \mathrm{CB}_{1}$ cannabinoid receptor \\ CBD cannabidiol \\ COVID-19 coronavirus disease 19 \\ GPCR G protein coupled receptor \\ MD molecular dynamics \\ MPR membrane-proximal region \\ NAM negative allosteric modulator
}


PAM

REST2

RMSD

RMSF

THC positive allosteric modulator

replica exchange. molecular dynamics with solute tempering

root mean square deviation

root mean square fluctuation

(-)-trans- $\Delta^{9}$-tetrahydrocannabinol

\section{References}

1. Howlett, A.C.; Breivogel, C.S.; Childers, S.R.; Deadwyler, S.A.; Hampson, R.E.; Porrino, L.J. Cannabinoid physiology and pharmacology: 30 years of progress. Neuropharmacology 2004, 47 (Suppl. 1), 345-358. [CrossRef]

2. Stasiulewicz, A.; Znajdek, K.; Grudzien, M.; Pawinski, T.; Sulkowska, A.J.I. A Guide to Targeting the Endocannabinoid System in Drug Design. Int. J. Mol. Sci. 2020, 21, 2778. [CrossRef]

3. Alaverdashvili, M.; Laprairie, R.B. The future of type 1 cannabinoid receptor allosteric ligands. Drug Metab. Rev. 2018, 50, 14-25. [CrossRef]

4. Lazzari, P.; Pau, A.; Tambaro, S.; Asproni, B.; Ruiu, S.; Pinna, G.; Mastinu, A.; Curzu, M.M.; Reali, R.; Bottazzi, M.E.; et al. Synthesis and pharmacological evaluation of novel 4-alkyl-5-thien-2'-yl pyrazole carboxamides. Cent. Nerv. Syst. Agents Med. Chem. 2012, 12, 254-276. [CrossRef]

5. Mastinu, A.; Pira, M.; Pani, L.; Pinna, G.A.; Lazzari, P. NESS038C6, a novel selective CB1 antagonist agent with anti-obesity activity and improved molecular profile. Behav. Brain Res. 2012, 234, 192-204. [CrossRef]

6. Lu, D.; Immadi, S.S.; Wu, Z.; Kendall, D.A. Translational potential of allosteric modulators targeting the cannabinoid CB1 receptor. Acta Pharmacol. Sin. 2019, 40, 324-335. [CrossRef]

7. Nguyen, T.; Li, J.X.; Thomas, B.F.; Wiley, J.L.; Kenakin, T.P.; Zhang, Y. Allosteric Modulation: An Alternate Approach Targeting the Cannabinoid CB1 Receptor. Med. Res. Rev. 2017, 37, 441-474. [CrossRef]

8. Price, M.R.; Baillie, G.L.; Thomas, A.; Stevenson, L.A.; Easson, M.; Goodwin, R.; McLean, A.; McIntosh, L.; Goodwin, G.; Walker, G.; et al. Allosteric modulation of the cannabinoid CB1 receptor. Mol. Pharmacol. 2005, 68, 1484-1495. [CrossRef]

9. Wootten, D.; Christopoulos, A.; Sexton, P.M. Emerging paradigms in GPCR allostery: Implications for drug discovery. Nat. Rev. Drug Discov. 2013, 12, 630-644. [CrossRef]

10. Laprairie, R.B.; Bagher, A.M.; Kelly, M.E.; Denovan-Wright, E.M. Cannabidiol is a negative allosteric modulator of the cannabinoid CB1 receptor. Br. J. Pharmacol. 2015, 172, 4790-4805. [CrossRef]

11. Bracey, M.H.; Hanson, M.A.; Masuda, K.R.; Stevens, R.C.; Cravatt, B.F. Structural adaptations in a membrane enzyme that terminates endocannabinoid signaling. Science 2002, 298, 1793-1796. [CrossRef] [PubMed]

12. Gado, F.; Meini, S.; Bertini, S.; Digiacomo, M.; Macchia, M.; Manera, C. Allosteric modulators targeting cannabinoid cb1 and cb2 receptors: Implications for drug discovery. Future Med. Chem. 2019, 11, 2019-2037. [CrossRef] [PubMed]

13. Gentry, P.R.; Sexton, P.M.; Christopoulos, A. Novel Allosteric Modulators of G Protein-coupled Receptors. J. Biol. Chem. 2015, 290, 19478-19488. [CrossRef] [PubMed]

14. Slivicki, R.A.; Xu, Z.; Kulkarni, P.M.; Pertwee, R.G.; Mackie, K.; Thakur, G.A.; Hohmann, A.G. Positive Allosteric Modulation of Cannabinoid Receptor Type 1 Suppresses Pathological Pain Without Producing Tolerance or Dependence. Biol. Psychiatry 2018, 84, 722-733. [CrossRef]

15. Hryhorowicz, S.; Kaczmarek-Rys, M.; Andrzejewska, A.; Staszak, K.; Hryhorowicz, M.; Korcz, A.; Slomski, R. Allosteric Modulation of Cannabinoid Receptor 1-Current Challenges and Future Opportunities. Int. J. Mol. Sci. 2019, 20, 5874. [CrossRef]

16. Christopoulos, A.; Kenakin, T. G protein-coupled receptor allosterism and complexing. Pharmacol. Rev. 2002, 54, 323-374. [CrossRef]

17. Morales, P.; Jagerovic, N. Novel approaches and current challenges with targeting the endocannabinoid system. Expert. Opin. Drug Discov. 2020, 15, 917-930. [CrossRef]

18. Mastinu, A.; Ribaudo, G.; Ongaro, A.; Bonini, S.A.; Memo, M.; Gianoncelli, A. Critical Review on the Chemical Aspects of Cannabidiol (CBD) and Harmonization of Computational Bioactivity Data. Curr. Med. Chem. 2021, 28, 213-237. [CrossRef]

19. Esposito, G.; Pesce, M.; Seguella, L.; Sanseverino, W.; Lu, J.; Corpetti, C.; Sarnelli, G. The potential of cannabidiol in the COVID-19 pandemic. Br. J. Pharmacol. 2020, 177, 4967-4970. [CrossRef]

20. Costiniuk, C.T.; Jenabian, M.A. Acute inflammation and pathogenesis of SARS-CoV-2 infection: Cannabidiol as a potential anti-inflammatory treatment? Cytokine Growth Factor Rev. 2020, 53, 63-65. [CrossRef]

21. Salles, E.L.; Khodadadi, H.; Jarrahi, A.; Ahluwalia, M.; Paffaro, V.A., Jr.; Costigliola, V.; Yu, J.C.; Hess, D.C.; Dhandapani, K.M.; Baban, B. Cannabidiol (CBD) modulation of apelin in acute respiratory distress syndrome. J. Cell. Mol. Med. 2020. [CrossRef]

22. Byrareddy, S.N.; Mohan, M. SARS-CoV2 induced respiratory distress: Can cannabinoids be added to anti-viral therapies to reduce lung inflammation? Brain. Behav. Immun. 2020, 87, 120-121. [CrossRef]

23. Huang, C.; Wang, Y.; Li, X.; Ren, L.; Zhao, J.; Hu, Y.; Zhang, L.; Fan, G.; Xu, J.; Gu, X.; et al. Clinical features of patients infected with 2019 novel coronavirus in Wuhan, China. Lancet 2020, 395, 497-506. [CrossRef]

24. Manuzak, J.A.; Gott, T.M.; Kirkwood, J.S.; Coronado, E.; Hensley-McBain, T.; Miller, C.; Cheu, R.K.; Collier, A.C.; Funderburg, N.T.; Martin, J.N.; et al. Heavy Cannabis Use Associated with Reduction in Activated and Inflammatory Immune Cell Frequencies in Antiretroviral Therapy-Treated Human Immunodeficiency Virus-Infected Individuals. Clin. Infect. Dis. 2018, 66, 1872-1882. [CrossRef] 
25. Wang, B.; Kovalchuk, A.; Li, D.; Ilnytskyy, Y.; Kovalchuk, I.; Kovalchuk, O. In Search of Preventative Strategies: Novel AntiInflammatory High-CBD Cannabis Sativa Extracts Modulate ACE2 Expression in COVID-19 Gateway Tissues. Preprints 2020. [CrossRef]

26. Russo, E.; Guy, G.W. A tale of two cannabinoids: The therapeutic rationale for combining tetrahydrocannabinol and cannabidiol. Med. Hypotheses 2006, 66, 234-246. [CrossRef] [PubMed]

27. Russo, E.B. Taming THC: Potential cannabis synergy and phytocannabinoid-terpenoid entourage effects. Br. J. Pharmacol. 2011, 163, 1344-1364. [CrossRef] [PubMed]

28. Pertwee, R.G.; Ross, R.A.; Craib, S.J.; Thomas, A. (-)-Cannabidiol antagonizes cannabinoid receptor agonists and noradrenaline in the mouse vas deferens. Eur. J. Pharmacol. 2002, 456, 99-106. [CrossRef]

29. Fay, J.F.; Farrens, D.L. The membrane proximal region of the cannabinoid receptor CB1 N-terminus can allosterically modulate ligand affinity. Biochemistry 2013, 52, 8286-8294. [CrossRef]

30. Baillie, G.L.; Horswill, J.G.; Anavi-Goffer, S.; Reggio, P.H.; Bolognini, D.; Abood, M.E.; McAllister, S.; Strange, P.G.; Stephens, G.J.; Pertwee, R.G.; et al. CB(1) receptor allosteric modulators display both agonist and signaling pathway specificity. Mol. Pharmacol. 2013, 83, 322-338. [CrossRef]

31. Sabatucci, A.; Tortolani, D.; Dainese, E.; Maccarrone, M. In silico mapping of allosteric ligand binding sites in type-1 cannabinoid receptor. Biotechnol. Appl. Biochem. 2018, 65, 21-28. [CrossRef] [PubMed]

32. Chung, H.; Fierro, A.; Pessoa-Mahana, C.D. Cannabidiol binding and negative allosteric modulation at the cannabinoid type 1 receptor in the presence of delta-9-tetrahydrocannabinol: An In Silico study. PLoS ONE 2019, 14, e0220025. [CrossRef] [PubMed]

33. Kooistra, A.J.; Mordalski, S.; Pandy-Szekeres, G.; Esguerra, M.; Mamyrbekov, A.; Munk, C.; Keseru, G.M.; Gloriam, D.E. GPCRdb in 2021: Integrating GPCR sequence, structure and function. Nucleic Acids Res. 2021, 49, D335-D343. [CrossRef] [PubMed]

34. Shao, Z.; Yin, J.; Chapman, K.; Grzemska, M.; Clark, L.; Wang, J.; Rosenbaum, D.M. High-resolution crystal structure of the human CB1 cannabinoid receptor. Nature 2016, 540, 602-606. [CrossRef]

35. Murphy, J.W.; Kendall, D.A. Integrity of extracellular loop 1 of the human cannabinoid receptor 1 is critical for high-affinity binding of the ligand CP 55,940 but not SR 141716A. Biochem. Pharmacol. 2003, 65, 1623-1631. [CrossRef]

36. Jakowiecki, J.; Orzel, U.; Chawananon, S.; Miszta, P.; Filipek, S. The Hydrophobic Ligands Entry and Exit from the GPCR Binding Site-SMD and SuMD Simulations. Molecules 2020, 25, 1930. [CrossRef]

37. Jo, S.; Jiang, W. A generic implementation of replica exchange with solute tempering (REST2) algorithm in NAMD for complex biophysical simulations. Comput. Phys. Commun. 2015, 197, 304-311. [CrossRef]

38. Filipek, S. Molecular switches in GPCRs. Curr. Opin. Struct. Biol. 2019, 55, 114-120. [CrossRef]

39. Yuan, S.; Hu, Z.; Filipek, S.; Vogel, H. W246(6.48) opens a gate for a continuous intrinsic water pathway during activation of the adenosine A2A receptor. Angew. Chem. Int. Ed. 2015, 54, 556-559. [CrossRef]

40. Yuan, S.; Peng, Q.; Palczewski, K.; Vogel, H.; Filipek, S. Mechanistic Studies on the Stereoselectivity of the Serotonin 5-HT1A Receptor. Angew. Chem. Int. Ed. 2016, 55, 8661-8665. [CrossRef]

41. Ballesteros, J.A.; Weinstein, H. Integrated methods for the construction of three-dimensional models and computational probing of structure-function relations in G protein-coupled receptors. Methods Neurosci. 1995, 25, 366-428.

42. Isberg, V.; de Graaf, C.; Bortolato, A.; Cherezov, V.; Katritch, V.; Marshall, F.H.; Mordalski, S.; Pin, J.P.; Stevens, R.C.; Vriend, G.; et al. Generic GPCR residue numbers-Aligning topology maps while minding the gaps. Trends Pharmacol. Sci. 2015, 36, 22-31. [CrossRef]

43. Krieger, E.; Vriend, G. New ways to boost molecular dynamics simulations. J. Comput. Chem. 2015, 36, 996-1007. [CrossRef]

44. Mandell, D.J.; Coutsias, E.A.; Kortemme, T. Sub-angstrom accuracy in protein loop reconstruction by robotics-inspired conformational sampling. Nat. Methods 2009, 6, 551-552. [CrossRef] [PubMed]

45. Salomon-Ferrer, R.; Case, D.A.; Walker, R.C. An overview of the Amber biomolecular simulation package. Wires Comput. Mol. Sci. 2013, 3, 198-210. [CrossRef]

46. UniProt, C. UniProt: A worldwide hub of protein knowledge. Nucleic Acids Res. 2019, 47, D506-D515. [CrossRef]

47. Canutescu, A.A.; Dunbrack, R.L., Jr. Cyclic coordinate descent: A robotics algorithm for protein loop closure. Protein Sci. 2003, 12, 963-972. [CrossRef]

48. Phillips, J.C.; Hardy, D.J.; Maia, J.D.C.; Stone, J.E.; Ribeiro, J.V.; Bernardi, R.C.; Buch, R.; Fiorin, G.; Henin, J.; Jiang, W.; et al. Scalable molecular dynamics on CPU and GPU architectures with NAMD. J. Chem. Phys. 2020, 153, 044130. [CrossRef] [PubMed]

49. Huang, J.; Rauscher, S.; Nawrocki, G.; Ran, T.; Feig, M.; de Groot, B.L.; Grubmuller, H.; MacKerell, A.D., Jr. CHARMM36m: An improved force field for folded and intrinsically disordered proteins. Nat. Methods 2017, 14, 71-73. [CrossRef]

50. Jo, S.; Kim, T.; Iyer, V.G.; Im, W. CHARMM-GUI: A web-based graphical user interface for CHARMM. J. Comput. Chem. 2008, 29, 1859-1865. [CrossRef]

51. Ryckaert, J.-P.; Ciccotti, G.; Berendsen, H.J. Numerical integration of the cartesian equations of motion of a system with constraints: Molecular dynamics of n-alkanes. J. Comput. Phys. 1977, 23, 327-341. [CrossRef]

52. Essmann, U.; Perera, L.; Berkowitz, M.L.; Darden, T.; Lee, H.; Pedersen, L.G. A Smooth Particle Mesh Ewald Method. J. Chem. Phys. 1995, 103, 8577-8593. [CrossRef]

53. Jones, G.; Willett, P.; Glen, R.C.; Leach, A.R.; Taylor, R. Development and validation of a genetic algorithm for flexible docking. J. Mol. Biol. 1997, 267, 727-748. [CrossRef] [PubMed] 
54. Lomize, M.A.; Pogozheva, I.D.; Joo, H.; Mosberg, H.I.; Lomize, A.L. OPM database and PPM web server: Resources for positioning of proteins in membranes. Nucleic Acids Res. 2012, 40, D370-D376. [CrossRef] [PubMed]

55. Miyamoto, S.; Kollman, P.A. SETTLE: An analytical version of the SHAKE and RATTLE algorithm for rigid water models. J. Comput. Chem. 1992, 13, 952-962. [CrossRef]

56. Roe, D.R.; Cheatham, T.E., 3rd. PTRAJ and CPPTRAJ: Software for Processing and Analysis of Molecular Dynamics Trajectory Data. J. Chem. Theory Comput. 2013, 9, 3084-3095. [CrossRef] 